\title{
Regularity of Conjugacies between Critical Circle Maps: An Experimental Study
}

\author{
Rafael de la Llave and Nikola P. Petrov
}

\section{CONTENTS}

1. Introduction

2. Rigorous Results about Conjugacies between Circle Maps

3. Some General Heuristic Remarks on Renormalization and Conjugacies

4. Computing the Conjugacies

5. Methods for Studying the Regularity

6. Numerical Implementation

7. Results

8. Some Bounds on the Regularity of Conjugacies

9. Conclusion

Acknowledgments

References
2000 AMS Subject Classification: Primary 37E10, 37-04, 34C41, 37F50, 37F 25

Keywords: Critical circle maps, self-similarity, renormalization, smoothness of conjugacies
We develop numerical implementations of several criteria to assess the regularity of functions. The criteria are based on the finite difference method and harmonic analysis: Littlewood-Paley theory, and wavelet analysis.

As a first application of the methods, we study the regularity of conjugacies between critical circle maps (i.e., differentiable homeomorphisms with a critical point) with a golden mean rotation number. These maps have a very well-developed mathematical theory as well as a wealth of numerical studies.

We compare the results produced by our methods among themselves and with theorems in the mathematical literature. We confirm that several of the features that are predicted by the mathematical results are observable by numerical computation. Some universal numbers predicted can be computed reliably. Our calculations suggest that several simple upper bounds are sharp in some cases, but not in others. This indicates that there may be conceptually different mechanisms at play.

\section{INTRODUCTION}

Classification of circle homeomorphisms under changes of variables is an old and famous problem in mathematics. It was initiated in [Poincaré 1885], motivated by studies in differential equations more than a century ago, and has been actively studied ever since.

Circle maps are also important because of their applications to natural sciences. They appear in the PomeauManneville scenario for transition to turbulence through intermittency [Pommeau and Manneville 80], second order ODEs with periodic potentials [Moser and Pöschel 84], cardiac arrhythmias [Glass 95], oscillations in plasma [Ignatov 95], electronic devices [Bohr et al. 84], and optical resonators with a periodically moving wall [de la Llave and Petrov 99], to name just a few. We would like to draw the reader's attention to the collections of reprints, [Cvitanović 89] and [Bai-Lin Hao 90], which contain many articles devoted to circle maps and their applications.

(c) A K Peters, Ltd 1058-6458/2001 \$0.50 per page Experimental Mathematics 11:2, page 219 
The main dynamical invariant of homeomorphisms of the circle is the rotation number (see Section 2.1). It was quickly realized that it is an invariant under topological equivalence [Poincaré 1885] and that for $C^{2}$ maps, it is an invariant for topological conjugacy [Denjoy 32]. The theory of smooth equivalence of smooth diffeomorphisms is now very well understood ([Herman 79], [Katznelson and Ornstein 89]).

Nevertheless, the theory of smooth equivalence of "critical circle maps," i.e., smooth circle maps that are homeomorphisms, but not diffeomorphisms (the simplest one - and the only one that we will consider in this paper-being a smooth map with a critical point), is much less developed. This will be the main subject of our empirical studies.

In the articles [Shenker 82], [Feigenbaum et al. 82], [Rand et al. 82], and [Ostlund et al. 83], it was found numerically that cubic critical circle maps exhibit interesting "universal" properties - for large classes of circle maps there exist numbers and functions that are the same for all functions in the class - similar to the FeigenbaumCoullet-Tresser universality of unimodal maps of the interval. (Lately, similar studies have been carried out for maps with critical points of higher degrees [Dixon et al. 97], [Briggs at al. 98]).

Shortly after the initial numerical studies, a renormalization theory that explains these properties was developed and some parts of the theory were given a firm mathematical basis [Feigenbaum et al. 82], [Ostlund et al. 83], [Shraiman 84], [Epstein 86], [Eckmann and Epstein 86], [Eckmann and Epstein 87], [Lanford 86], [Lanford 87], [Lanford 88], [Rand 87], [Rand 88a], [Rand 88b], [Kim and Ostlund 89], [Epstein 89], [Veerman and Tangerman 90], [Tangerman and Veerman 91], [Pinto and Rand 92], [Rand 92]. Recently there has been significant progress in the renormalization theory of critical circle maps [Świa̧tek 98], [de Melo 98], [Yampolsky 99], [de Faria 99], [de Faria and de Melo 99], [de Faria and de Melo 00].

In [Shenker 82], a one-parameter family of smooth circle maps, $\left\{f_{K}\right\}$, all of rotation number equal to the golden mean, $\gamma=\frac{\sqrt{5}-1}{2}$, was studied numerically. It was found that if $f_{K}$ is a diffeomorphism, the conjugacy between $f_{K}$ and the rotation by $\gamma$ is a smooth function (as predicted by the general theory) [Herman 79]. However, if $f_{K}$ has a cubic critical point, the conjugacy between $f_{K}$ and the rotation becomes very rough. Moreover, it was noticed that the conjugacy has a self-similar structure (which was found by studying the first 400 Fourier coefficients of the conjugacy).
In the present paper, we study numerically the smoothness of the conjugacies between noncritical, cubic critical, and quintic critical circle maps. To estimate the smoothness of the conjugacies, we use finite difference methods and tools from harmonic analysis (LittlewoodPaley theory and wavelet theory). In some cases, we are able to reliably calculate millions of Fourier coefficients, so we hope that our numerical estimates are convincing.

We expect that the numerical methodology developed here will be used to study several other problems in the theory of critical phenomena in dynamical systems in which the regularity of functions and their self-similar properties play a role. Since the theory of circle maps has a well-developed mathematical literature, it seemed a good starting point to assess the validity of the methods.

We also find evidence that the conjugating functions are asymptotically self-similar, confirming by very different methods the results obtained previously for cubic critical maps. (See the numerical studies in [Arneodo and Holschneider 88].)

The fact that we have precise numbers for the regularity of the conjugacies predicted in the theorems allows us to observe that some simple upper bounds for the regularity of the conjugacies appear to be sharp, whereas in other cases, they seem to be very far from optimal. This indicates the possibility of conceptually different phenomena at play. (See Section 8..)

\section{RIGOROUS RESULTS ABOUT CONJUGACIES BETWEEN CIRCLE MAPS}

In this section we briefly recall some basic definitions from the theory of circle maps (for details see, e.g., [Katok and Hasselblatt 95], [de Melo and Van Strien 98], and [Herman 79]). We also collect some recent results in the mathematical literature. We cannot aim for completeness, but just want to set the notation and to give the reader a feeling for the results.

\subsection{Rotation Number}

If $F: \mathbb{R} \rightarrow \mathbb{R}$ is a map satisfying $F(x+1)=F(x)+1$, and if

$$
\pi: \mathbb{R} \rightarrow \mathbb{T} \equiv \mathbb{R} / \mathbb{Z}: x \mapsto \pi(x):=x \quad \bmod 1,
$$

then the map

$$
f:=\pi \circ F: \mathbb{T} \rightarrow \mathbb{T}
$$

is a map of the circle $\mathbb{T}$, and $F$ is called a lift of $f$. In this paper, a "circle map" will always mean orientationpreserving circle homeomorphism. 
The most important characteristic for the classification of circle maps is the rotation number. If $f: \mathbb{T} \rightarrow \mathbb{T}$ is a circle homeomorphism and $F$ is a lift of $f$, then the rotation number $\tau(f)$ of $f$ is defined by

$$
\tau(f):=\left(\lim _{n \rightarrow \infty} \frac{F^{n}(x)-x}{n}\right) \bmod 1 .
$$

The above limit exists and is independent of the choice of the lift and the point $x \in \mathbb{T}$.

The simplest circle map is the rotation,

$$
r_{\rho}: \mathbb{T} \rightarrow \mathbb{T}: x \mapsto(x+\rho) \bmod 1,
$$

whose rotation number is obviously $\tau\left(r_{\rho}\right)=\rho \bmod 1$. For $r_{\rho}$, there are two types of orbits of the points of $\mathbb{T}$. If $\rho=p / q \in \mathbb{Q}$ (with $p$ and $q$ relatively prime), then the orbit $\left\{r_{\rho}^{n}(x)\right\}_{n \in \mathbb{Z}}$ is periodic of minimal period $q$. If $\rho \notin \mathbb{Q}$, then there are no periodic orbits for the rotation $r_{\rho}$ and the orbit of any point $x \in \mathbb{T}$ is dense in $\mathbb{T}$.

\subsection{Conjugacies: Existence Theorems}

Because of the simplicity of the rotations, it is natural to investigate whether a particular circle map $f$ is "equivalent" in some sense to a rotation, i.e., whether there exists a change of variables $y=h(x)$ such that in the new variables, $f$ "looks like" a rotation.

Definition 2.1. Two circle maps $f$ and $g$ are topologically (respectively $C^{k}$-, smoothly, analytically) conjugate if there exists a homeomorphism (respectively $C^{k}, C^{\infty}$, or $C^{\omega}$ diffeomorphism) $h: \mathbb{T} \rightarrow \mathbb{T}$ such that

$$
f=h^{-1} \circ g \circ h .
$$

The map $h$ is called the conjugacy between $f$ and $g$.

If $f$ and $g$ are conjugate, many of their properties are the same - e.g., the possible types of the orbits of the points of $\mathbb{T}$ under $f$ and under $g$ are the same, the rotation number of $f$ is equal to that of $g$, etc.

Early results about the existence of a conjugacy to a rotation can be found in the following theorems [Poincaré 1885], [Denjoy 32].

Theorem 2.2. (Poincaré.) Assume that the rotation number of the circle map $f$ is irrational and the orbit of some point $x \in \mathbb{T}$ is dense in $\mathbb{T}$. Then $f$ is topologically conjugate to $r_{\tau(f)}$. (If only the first condition is satisfied, then there exists a continuous map $h: \mathbb{T} \rightarrow \mathbb{T}$ such that $\left.h \circ f=r_{\tau(f)} \circ h.\right)$
Theorem 2.3. (Denjoy.) A circle diffeomorphism with irrational rotation number and derivative of bounded variation is topologically conjugate to a rotation.

Denjoy's theorem implies that every $C^{2}$ circle diffeomorphism with irrational rotation number is topologically conjugate to a rotation. The $C^{2}$ condition is rather sharp-Herman [Herman 79, Section X.3.19] constructed examples of $C^{2-\varepsilon}$ circle diffeomorphisms that are not conjugate to a rotation, even if the rotation number satisfies additional restrictions. However, a proof of Denjoy's theorem for $f$, a diffeomorphism in $\Lambda_{2}$ (see Definition 5.1), is given in [Hu and Sullivan 97]. (Note that the derivative of a $\Lambda_{2}$ function could fail to have bounded variation.)

In Denjoy's theorem, it is important that $f^{-1}$ is differentiable. If $f^{-1}$ is not differentiable, one cannot guarantee the existence of a conjugacy to a rotation even by assuming that $f$ is $C^{\infty}$. The article by Hall [Hall 81] contains an example of a $C^{\infty}$ circle map with rotation number $\rho$ (for any irrational $\rho \in[0,1)$ ) which has no dense orbit and therefore cannot be conjugate to $r_{\rho}$. This map is onto, has no periodic orbits, and has no more than two points where the derivative of the map vanishes (in fact, one can construct such a map with only one critical point).

In striking contrast to the $C^{\infty}$ Denjoy counterexamples of [Hall 81], it was shown [Yoccoz 84a] that the maps exhibiting the behavior of those in [Hall 81] cannot be real analytic. More precisely:

Theorem 2.4. (Yoccoz.) Any real analytic circle map with no periodic orbits is topologically conjugate to a rotation.

This theorem guarantees that any two real analytic circle maps with irrational rotation numbers are topologically conjugate (we will use this fact in Section 4.3). The above result was extended in [Świątek 91], and [Świạtek 98].

\subsection{Smoothness of the Conjugacy}

The theorems in Section 2.2 do not assert anything about the smoothness of the conjugacy $h$. Can one say more about the differentiability properties of the conjugacies in the case of smooth or analytic maps? The answer to this question involves number-theoretic properties of the rotation number.

2.3.1 Arithmetic Properties of the Rotation Number. It turns out that, to guarantee that a smooth circle diffeomorphism $f$ is smoothly conjugate to a rotation, it is 
not enough that $\tau(f)$ be irrational; it also should not be well approximable by rationals.

Definition 2.5. A number $\rho$ is called Diophantine of type $(C, \nu)$ (or simply of type $\nu$ ) for positive $C$ and $\nu$, if for any $p / q \in \mathbb{Q}$

$$
\left|\rho-\frac{p}{q}\right|>\frac{C}{|q|^{2+\nu}} .
$$

A number that is not Diophantine is called a Liouville number.

It is easy to show that there are no numbers that satisfy $(2-3)$ when $\nu<0$. Hence, we will assume $\nu \geq 0$.

Each irrational number $\rho \in(0,1)$ can be written in the continued fraction representation as

$$
\rho=\left\langle\rho_{0}, \rho_{1}, \rho_{2}, \ldots\right\rangle:=\frac{1}{\rho_{0}+\frac{1}{\rho_{1}+\frac{1}{\rho_{2}+\cdots}}},
$$

where the positive integers $\rho_{n}$ are called partial quotients of $\rho$.

In this representation, a number is Diophantine if its partial quotients increase slowly. In this sense, the "most irrational" number between 0 and 1 is the golden mean,

$$
\gamma:=\langle 1,1,1, \ldots\rangle=\frac{\sqrt{5}-1}{2}=\lim _{n \rightarrow \infty} \frac{Q_{n}}{Q_{n+1}},
$$

where $Q_{1}=1, Q_{2}=2, Q_{n+1}=Q_{n}+Q_{n-1}$ are the Fibonacci numbers.

When the partial quotients of $\rho$ are bounded, $\rho$ is called a number of bounded (or constant) type. This is equivalent to the fact that $(2-3)$ holds with $\nu=0$. The combinatorial type of $\rho$ is by definition $\sup _{n} \rho_{n}$.

2.3.2 Results for Noncritical Maps. The question about the smoothness of the conjugacy in the case of analytic circle maps close to a rotation was first addressed in [Arnold 61]. There, it was proved that any analytic circle map whose rotation number $\rho$ is Diophantine of type $\nu \geq 0$, and which is close to a rotation, is analytically conjugate to $r_{\rho}$. This result was extended to the case of finite differentiability in [Moser 66].

The first theorem without the hypothesis of closeness to a rotation was proved in [Herman 79]. The theorem states that there exists a set $\mathcal{K} \subset[0,1]$ of full Lebesgue measure such that if $f \in C^{k}$ for $3 \leq k \leq \omega$ and $\tau(f) \in \mathcal{K}$, then the conjugacy is $C^{k-2-\varepsilon}$ for any $\varepsilon>0$. The set $\mathcal{K}$ is characterized in terms of the growth of the partial quotients of the continued fraction expansions of its members; all numbers in $\mathcal{K}$ are Diophantine of order $\nu$ for any $\nu>0$. This result was significantly improved in [Yoccoz 84b]. Similar theorems were proved by using renormalization group techniques [Sinai and Khanin 89], which lowered the minimal regularity required. The most comprehensive result we have found in the literature is the one in [Katznelson and Ornstein 89].

Theorem 2.6. If $f$ is a $C^{k}$ circle diffeomorphism whose rotation number is Diophantine of order $\nu$, and $k>\nu+2$, then the homeomorphism $h$ that conjugates $f$ with the rotation $r_{\tau(f)}$ is of class $C^{k-1-\nu-\varepsilon}$ for any $\varepsilon>0$.

2.3.3 Results for Critical Maps. We recall that a crit$i c a l$ circle map is a circle homeomorphism whose derivative is positive except at one point where it is a zero of finite order. The order of the zero is called the type of the critical map.

The following result was proved recently ([de Faria and de Melo 99], [de Faria and de Melo 00]; see also [de Melo 98], [de Faria 99], and [Yampolsky 99]):

Theorem 2.7. Let $f$ and $g$ be real analytic critical circle maps with the same type of the critical point and with the same rotation number $\rho$ of bounded type. Then $f$ and $g$ are $C^{1+\alpha}$ conjugate for some $\alpha \in(0,1)$ depending only on the combinatorial type, $N:=\sup _{n} \rho_{n}$, of $\rho$.

It was conjectured in [de Melo 98] that the Hölder exponent of the conjugacy between two critical circle maps whose critical points are of the same order and which have the same rotation number of bounded type does not depend on $N$ (although in the proof of Theorem 2.7 $\alpha$ does depend on $N)$.

For the regularity of the conjugacies between critical maps and rotations, which exist because of Theorem 2.4 , the sharpest result of which we are aware is from [Światek 98], whose Theorem 1.1 (the theorem and a previous proof are credited to an unpublished manuscript of M. Herman) implies:

Theorem 2.8. Let $f$ be an analytic critical circle map with an irrational rotation number. The conjugacy between the map and a rotation is quasi-symmetric if and only if the rotation number is of constant type.

We note that it is a well-known fact in the theory of quasi-conformal maps that the quasi-symmetric maps are Hölder ([Väisälä 71, Section 18], [Ghering and Palka, $\mathrm{xx}]$ ). Hence, we can conclude that the conjugacy between 
a critical map and a rotation is Hölder. Therefore, the conjugacies between critical circle maps of the same rotation number have to be Hölder with exponent 1 . It is not difficult to show that, when the critical maps have different order, the conjugacy between them cannot have Hölder exponent 1.

Putting together Theorem 2.8, the Hölder regularity of quasi-symmetric maps and Theorem 2.7, we obtain:

Corollary 2.9. The conjugacy between critical circle maps with a golden mean rotation number is Hölder. The Hölder exponent depends only on the order of the critical points of the two functions.

The above results seem to give very little information about what the actual values of these regularities are. In this paper, we will develop methods that allow us to compute these numbers as well as to explore numerically some geometric properties of the conjugacies.

\section{SOME GENERAL HEURISTIC REMARKS ON RENORMALIZATION AND CONJUGACIES}

A unifying point of view in the study of long term dynamics - especially in one-dimensional systems-has been provided by scaling and renormalization group ideas. Formulated somewhat loosely, the unifying idea of a renormalization group says that "highly iterated maps, when observed in small scales, have forms that are largely independent of the map" (The universal properties can be different in sets of maps of positive codimension.).

In this section, we present a heuristic point of view on the relation between the asymptotics of the renormalization group and the smoothness of the conjugacy which seems to be applicable to a wide variety of models.

The main connection between the study of regularity properties of conjugacies and renormalization groups arises from the fact that the regularity of the conjugacies is a very good test of universality properties.

Note that regularity depends on very fine scales. Moreover, Formula (4-8), which we will establish later, makes it clear that the conjugacies in increasingly smaller scales are determined by the increasingly longer recurrence times.

The fact that some conjugacies examined in very small scales are self-similar leads, at least in an informal way, to several consequences of universality for the conjugacies that we will explore empirically in the rest of the paper.

(A1) The regularity of the conjugacies between maps of the same universality class is a "universal number."
Note that for the case of golden mean circle maps, this is a consequence of Corollary 2.9.

(A2) These universal regularities of the conjugacies between maps in the same class are higher than those between maps of different classes.

Note that for circle maps, this follows from the observation that critical circle maps can be only Hölder of exponent less than one conjugate to maps with critical points of different order. By Theorem 2.7, the conjugacies between maps of the same order is $C^{1+\alpha}$.

(A3) The functions giving the conjugacies are asymptotically self-similar.

If $h_{1}$ and $h_{2}$ are conjugacies of maps $f_{1}, f_{2}$ to the golden mean rotation, (i.e., $f_{1} \circ h_{1}=h_{1} \circ r_{\gamma}, f_{2} \circ h_{2}=h_{2} \circ r_{\gamma}$ ), then $f_{1} \circ h_{1} \circ h_{2}^{-1}=h_{1} \circ h_{2}^{-1} \circ f_{2}$. The fact that we observe that $h_{1} \circ h_{2}^{-1}=k, h_{1}^{-1} \circ h_{2}=\ell$ are very smooth means that $h_{1}=k \circ h_{2}, h_{1}=h_{2} \circ \ell^{-1}$; in other words, we can obtain $h_{1}$ from $h_{2}$ by composition with a very smooth map. This makes the notion precise that $h_{1}$ and $h_{2}$ are very similar. Even if each of them is rather rough, the roughness of one is very precisely comparable to that of the other.

We hope that the present work serves as a stimulus for further mathematical investigations. Our calculations are precise enough that we can even study the corrections to (A3). We formulate them as:

(A4) The convergence to self-similarity is exponentially fast.

Somewhat more precisely (but still very far from a mathematically rigorous statement), we can write conjugacies in the form

$$
h(x)=\sum_{n} \lambda_{1}^{n} H_{1}\left(\alpha^{n} x\right)+\sum_{n} \lambda_{2}^{n} H_{2}\left(\alpha^{n} x\right)+\cdots
$$

for some $|\alpha|>1,1>\lambda_{1}>\lambda_{2}>\cdots$, where $\mu, \lambda_{1}, \lambda_{2}, \ldots$ are universal numbers.

Of course, scalings such as those in (3-1) do not, strictly speaking, make sense in the case where the variables are in the circle. Nevertheless, since (3-1) is supposed to hold in the asymptotic sense of the very small scales, we can identify the whole circle with the real line.

We also note that the following conjecture seems to be reasonable for many areas in which renormalization applies. The case of period doubling is studied extensively in [de la Llave and Schafer, 96]. 
Conjecture 3.1. Consider the sets $\mathcal{M}_{\nu}$ (for odd integer $\nu \geq 1$ ) of analytic maps of the circle such that:

(i) The maps are homeomorphisms.

(ii) They are of the form $f(x)=A x^{\nu}+O\left(x^{\nu+1}\right)$ with $A$ a nonzero constant (hence $f$ is a homeomorphism but not a diffeomorphism).

(iii) Their rotation number is the golden mean $\gamma$.

The sets $\mathcal{M}_{\nu}$ are manifolds. Then in an open set $\mathcal{B}_{\nu} \in$ $\mathcal{M}_{\nu}$, we can find foliations $\mathcal{F}^{i}$, integers $d_{i}$, and numbers $\lambda_{i}\left(d_{i}>d_{i+1}, \lambda_{i}<\lambda_{i+1}, \lambda_{i} \rightarrow \infty\right)$ such that, if $\mathcal{W}_{f}^{i}$ is the leaf of $\mathcal{F}^{i}$ passing through the map $f$, then:

(a) $\mathcal{W}_{f}^{i} \subset \mathcal{W}_{f}^{i+1}$.

(b) $\mathcal{W}_{f}^{i}$ is an analytic submanifold of $\mathcal{M}_{\nu}$ of codimension $d_{i}$.

(c) The foliations $\mathcal{F}^{i}$ are Hölder (i.e., the $\infty$-jets of the leaves $\mathcal{W}_{f}^{i}$ are Hölder with respect to $f$ ).

(d) if $f \in \mathcal{W}_{g}^{i}$, then $f$ is $C^{\lambda_{i}-\varepsilon}$ conjugate to $g$ for each $\varepsilon>0$.

(e) If $f \notin \mathcal{W}_{g}^{i}$, then $f$ is not $C^{\lambda_{i}+\varepsilon}$ conjugate to $g$ for each $\varepsilon>0$.

The most important consequence of this conjecture is that the conjugacies between maps in the classes $\mathcal{M}_{\nu}$ can only have regularities which belong to a discrete set $\left\{\lambda_{i}\right\}$ (ignoring the $\varepsilon$ 's which are as small as desired). In particular, if we know that a conjugacy is $C^{\lambda_{i}+\varepsilon}$, we can conclude that it is $C^{\lambda_{i+1}-\varepsilon}$.

In the rest of the paper, we will present methods that allow us to carry out high precision calculation of golden mean circle maps as well as an array of methods that asses the regularity of the conjugacies between them. By comparing the results of these different methods among themselves and with the results in the mathematical literature, we can assess their validity, and we hope to apply them in other contexts.

In the process of doing that, we also obtain some information about the relation between regularity and renormalization. In particular, in Section 8., we obtain indications that the regularities may be limited by other mechanisms than just simple scaling phenomena.

\section{COMPUTING THE CONJUGACIES}

\subsection{Examples}

Let $f$ and $g$ be analytic circle maps whose derivatives possibly vanish at one point; without loss of generality, we can take this point to be $x=0$. We studied numerically the following families of analytic circle maps (for values of $K$ for which the maps are invertible):

(i) the noncritical (N) family $(0 \leq K<1)$

$$
f_{K, \omega}^{\mathrm{N}}(x)=\left(x+\omega-\frac{K}{2 \pi} \sin 2 \pi x\right) \quad \bmod 1 ;
$$

(ii) the cubic critical (C) family $\left(0 \leq K<\frac{4}{3}\right)$

$$
\begin{aligned}
f_{K, \omega}^{\mathrm{C}}(x)=[x & +\omega-\frac{1}{2 \pi}(K \sin 2 \pi x \\
& \left.\left.+\frac{1-K}{2} \sin 4 \pi x\right)\right] \bmod 1,
\end{aligned}
$$

where the coefficients are chosen in such a way that for every $K$,

$$
f_{K, \omega}^{\mathrm{C}}(x)=\omega+\frac{2 \pi^{2}(4-3 K)}{3} x^{3}+O\left(x^{5}\right) ;
$$

(iii) the quintic critical (Q) family $\left(\frac{1}{2} \leq K<\frac{3}{2}\right)$

$$
\begin{aligned}
f_{K, \omega}^{\mathrm{Q}}(x)=[x & +\omega-\frac{1}{2 \pi}\left(K \sin 2 \pi x+\frac{9-8 K}{10} \sin 4 \pi x\right. \\
& \left.\left.+\frac{3 K-4}{15} \sin 6 \pi x\right)\right] \quad \bmod 1,
\end{aligned}
$$

where the coefficients are chosen in such a way that for every $K$,

$$
f_{K, \omega}^{\mathrm{Q}}(x)=\omega+\frac{8 \pi^{4}(3-2 K)}{5} x^{5}+O\left(x^{7}\right) .
$$

Of course, there are similar formulae for higher order critical points, but the calculations cannot be carried out easily.

We studied the case of rotation number equal to the golden mean $\gamma$,

$$
\left.\tau\left(f_{K, \omega}^{\bullet}\right)\right|_{\omega=\Omega \bullet(K)}=\gamma,
$$

where $\bullet$ stands for $\mathrm{N}, \mathrm{C}$, or Q. The golden mean is chosen because its continued fraction expansion is periodic (and simple). Hence, renormalization arguments can be expressed in terms of operators and, since all partial quotients of $\gamma$ are 1 , renormalization operators are as simple as possible.

Since we have to iterate $f_{K, \omega}^{\bullet}$, we need to know the value of $\Omega^{\bullet}(K)$ with a very high precision. We used the $\mathrm{C}++$ software package doubledouble [Briggs 00] which allowed us to use about 30 decimal places floating point precision arithmetic and to find $24-25$ digits of the parameter $\Omega^{\bullet}(K)$ in the case of $\mathrm{N}$ circle maps and about 16 digits of $\Omega^{\bullet}(K)$ in the $\mathrm{C}$ and $\mathrm{Q}$ cases.

To double check the results, we also used the GNU MP library [GMP 00] - a public domain library for arbitrary precision arithmetic. We wrote subroutines for 
high precision trigonometric functions using local Taylor series expansion, and tested them by using the following elegant and numerically stable method:

For $x, y$ positive, define $x_{1}:=x, y_{1}:=y$, and

$$
x_{n+1}:=\frac{x_{n}+y_{n}}{2}, \quad y_{n+1}:=\sqrt{x_{n} y_{n}}
$$

for $n \in \mathbb{N}$. The sequences $\left\{x_{n}\right\}$ and $\left\{y_{n}\right\}$ converge quadratically to a common limit called the ArithmeticGeometric Mean (AGM) of $x$ and $y, \mathrm{M}(x, y)$ (see, e.g., [Borwein and Borwein 87]). The AGM has many remarkable properties, e.g.,

$$
\frac{2}{\pi} \int_{0}^{\pi / 2} \frac{d \zeta}{\sqrt{x^{2} \cos ^{2} \zeta+y^{2} \sin ^{2} \zeta}}=\frac{1}{\mathrm{M}(x, y)}
$$

(in particular, elliptic integrals can be calculated using the AGM). To calculate precisely trigonometric functions, e.g., sin, one can use the fact that

$$
\arcsin x=\frac{x}{\mathrm{M}\left(\sqrt{1-x^{2}}, 1\right)},
$$

and calculate sin as the inverse function by using the program zeroin (which is quadratically convergent, so each iteration doubles the number of correct digits).

The AGM has been used for precise computations, in [Brent 76]; see also the discussion in [Borwein and Borwein 84] (both papers reprinted in [Berggren et al. 00]).

\subsection{Calculating Parameters for the Rotation Number of the Golden Mean}

Having chosen some $K$ (for which $f_{K, \omega}^{\bullet}$ is a homeomorphism), we first have to determine the value $\Omega^{\bullet}(K)$ such that (4-4) is satisfied. To achieve this, we use the following method [Greene 79], [Shenker 82]: First we determine the phase-locking intervals

$$
I_{K, n}^{\bullet}:=\left\{\omega \in[0,1] \mid \tau\left(f_{K, \omega}^{\bullet}\right)=\frac{Q_{n}}{Q_{n+1}}\right\}, \quad n \in \mathbb{N} .
$$

It is guaranteed that the value $\Omega^{\bullet}(K)$ we are looking for is between $I_{K, n}^{\bullet}$ and $I_{K, n+1}^{\bullet}$. If $\Omega_{n}^{\bullet}(K)$ is the end of $I_{K, n}^{\bullet}$ that is closer to $I_{K, n+1}^{\bullet}$, then we assume that

$$
\Omega_{n}^{\bullet}(K)=\Omega^{\bullet}(K)+C \beta_{\bullet}^{n},
$$

for some constants $-1<\beta_{\bullet}<0$ and $C$ ( $\beta_{\bullet}$ is a universal number that depends only on the degree of the critical point, while $C$ is different for different maps), and find $\Omega^{\bullet}(K)$ by Aitken extrapolation [Press et al. 92]. This assumption does not affect the validity of our results, and it speeds up our searches since the rotation numbers of fewer maps are computed. We note that the renormalization group picture also predicts $(4-5)$. Moreover, this assumption is in excellent agreement with our computations:

(i) in the $\mathrm{N}$ case, we find

$$
\beta_{\mathrm{N}}=-0.381966011250 \pm 10^{-12}=-\gamma^{\kappa_{\mathrm{N}}}
$$

$$
\text { for }\left|\kappa_{\mathrm{N}}-2\right|<6 \times 10^{-12} \text { (according to Theorem 2.6, }
$$
$\left.\kappa_{\mathrm{N}}=2\right)$;

(ii) in the $\mathrm{C}$ case, we find

$$
\beta_{\mathrm{C}}=-0.3529067 \pm 10^{-7}=-\gamma^{\kappa_{\mathrm{C}}}
$$$$
\text { for } \kappa_{\mathrm{C}}=2.1644347 \pm 0.0000006 \text {; }
$$

(iii) in the $\mathrm{Q}$ case, we find

$$
\beta_{\mathrm{Q}}=-0.32858 \pm 10^{-5}=-\gamma^{\kappa_{\mathrm{Q}}}
$$

for $\kappa_{\mathrm{Q}}=2.31286 \pm 0.00006$.

The values we found are in perfect agreement with those found in [Shenker 82] (for the $\mathrm{C}$ case), and, for different families of circle maps, in [Hu et al. 90], and [Delbourgo and Kenny 91]. Note that what we call $\beta$ is called $\delta^{-1}$ in these papers .

To find the phase-locking interval $I_{K, n}^{\bullet}$, we used the fact that when $\omega$ enters this interval, the map

$$
x \mapsto\left(F_{K, \omega}^{\bullet}\right)^{Q_{n+1}}(x)-Q_{n}
$$

(where $F_{K, \omega}^{\bullet}$ is the lift of $f_{K, \omega}^{\bullet}$, i.e., it is given by the same formula as $f_{K, \omega}^{\bullet}$, but without the $\bmod 1$ ) undergoes a tangent bifurcation. To determine the values at which bifurcations occur, we used the subroutines fmin and zeroin from [Forsythe et al. 77] (translated into $C$ and slightly modified).

We will denote by $f_{K}^{\bullet}$ the map $f_{K, \Omega}^{\bullet}(K)$ for $\Omega^{\bullet}(K)$ such that $\tau\left(f_{K}^{\bullet}\right)=\gamma$. In Table 1 , we give the values of $\Omega^{\bullet}(K)$ for the values of $K$ we studied numerically.

\subsection{Calculating the Conjugacies on an Equidistant Grid}

Having found appropriate values of the parameters of the maps $f$ and $g$ such that $\tau(f)=\tau(g)=\gamma$, we construct numerically the conjugacy $h$ between them. Instead of $h$, it is more convenient to study

$$
\theta:=h-\mathrm{Id}
$$

because $\theta$ is a periodic function; hence it is better suited for harmonic analysis. For brevity, we will denote the 


\begin{tabular}{|c|c|}
\hline Map & $\Omega^{\bullet}(K)$ \\
\hline$f_{0.2}^{\mathrm{N}}$ & 0.617425455584922780978570 \\
\hline$f_{0.3}^{\mathrm{N}}$ & 0.6166923606057855021928 \\
\hline$f_{0.5}^{\mathrm{N}}$ & 0.6145263876774487765559862 \\
\hline$f_{0.8}^{\mathrm{N}}$ & 0.61007440530846512053842071 \\
\hline$f_{0.3}^{\mathrm{C}}$ & 0.626871059546737818 \\
\hline$f_{0.6}^{\mathrm{C}}$ & 0.617607758640542315 \\
\hline$f_{0.7}^{\mathrm{C}}$ & 0.6148131852529150525 \\
\hline$f_{1.0}^{\mathrm{C}}$ & 0.606661063470112017 \\
\hline$f_{0.6}^{\mathrm{Q}}$ & 0.633133040895040332 \\
\hline$f_{0.9}^{\mathrm{Q}}$ & 0.616330501795706578 \\
\hline$f_{1.2}^{\mathrm{Q}}$ & 0.60250115301615805 \\
\hline$f_{4 / 3}^{\mathrm{Q}}$ & 0.59694625982733198 \\
\hline
\end{tabular}

TABLE 1. Values of $\Omega^{\bullet}(K)$ such that $\tau\left(f_{K}^{\bullet}\right)=\gamma$.

map $\theta$ defined by (4-6) for $h$ being a conjugacy between, say, an $\mathrm{N}$ map $f$ and a $\mathrm{C}$ map $g$ by $\theta^{\mathrm{NC}}$, and will call $\theta$ a "conjugacy."

From (2-2) we obtain

$$
h \circ f^{n}=g^{n} \circ h .
$$

Theorem 2.4, which guarantees the existence of the conjugacies between $f$ (resp., $g$ ) and the rotation $r_{\gamma}$, allows us to impose the condition $h(0)=0$ or, equivalently, $\theta(0)=0$. This implies that $\theta\left(f^{n}(0)\right)=g^{n}(0)-f^{n}(0)$, so the points

$$
\left(f^{n}(0), g^{n}(0)-f^{n}(0)\right)
$$

belong to the graph of $\theta$ and fill it densely. It is apparent that one can compute the points in $(4-8)$ by iterating $f$ and $g$ on 0 .

One problem with this calculation is that the points in (4-8) do not have first coordinates that are distributed on a equidistant grid, and to apply fast Fourier of wavelet transforms, we need to know the values of $\theta$ on an equidistant grid. We used the grid

$$
x_{\ell}:=2^{-L} \ell, \quad \ell=0,1, \ldots, 2^{L}-1
$$

for some $L \in \mathbb{N}$ (typically about 20). Since the iterates $\left\{f^{n}(0)\right\}$ are not equidistantly distributed, we used interpolation and calculated the values of the interpolating function at the points $\left\{x_{\ell}\right\}_{\ell=0}^{2^{L}-1}$. To this end, we used the cubic interpolation subroutines spline and seval from [Forsythe et al. 77] (the periodicity of $\theta$ was taken into account).

A major source of difficulty for the numerical computation is the fact that the iterates of a $\mathrm{C}$ or $\mathrm{Q}$ map are very nonuniformly distributed. To illustrate the seriousness of the problem, we show (in Figure 1) the distribution of four million iterates of $f_{0.6}^{\mathrm{Q}}$ and, for comparison,

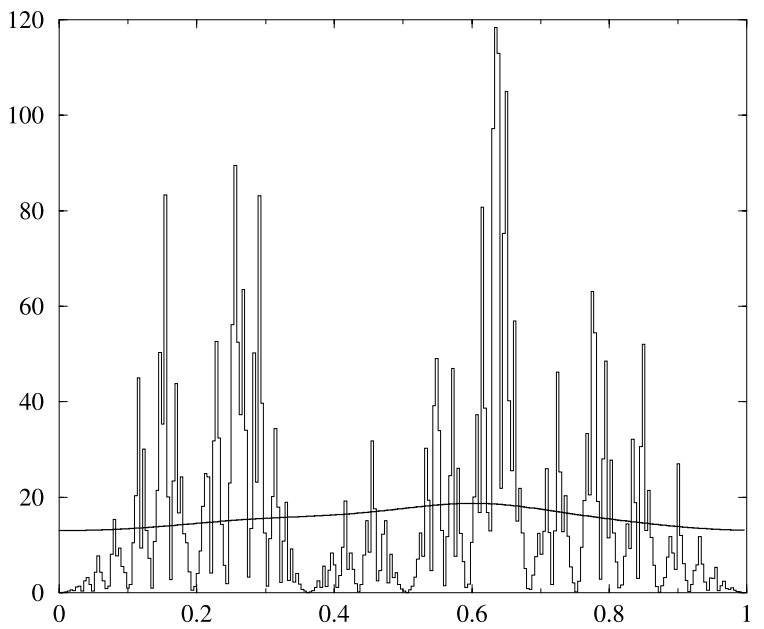

FIGURE 1. Density of the iterates of a $\mathrm{Q}$ and an $\mathrm{N}$ map. The number of iterates in a bin (in thousands) vs. the position of the bin, for four million iterates of $f_{0.6}^{\mathrm{Q}}$ (thin line) and of $f_{0.5}^{\mathrm{N}}$ (thick line), in 256 bins.

of $f_{0.3}^{\mathrm{N}}$, in 256 bins, each of size $\frac{1}{256}$, between 0 and 1 . The number of iterates of $f_{0.6}^{\mathrm{Q}}$ in a bin varies from 15 to 118304 ; for $f_{0.5}^{\mathrm{N}}$, it varies from 13076 to 18739 . The largest gap between the iterates of $f_{0.6}^{\mathrm{Q}}$ is 0.001308 .

If the gaps are very large, it is complicated and unstable to compute the values of the interpolating function at the gaps. We dealt with this by using a large number of iterates which is, however, very memory-consuming and leads to accumulation of numerical error. This problem becomes more severe when the order of the critical point is higher. This is the main reason why our investigation did not cover critical maps of degree higher than 5 .

\subsection{Conjugacies-Visual Explorations}

Theorem 2.6 guarantees that each $\theta^{\mathrm{NN}}$ (recall that this means a conjugacy between two $\mathrm{N}$ circle maps) is analytic, but does not say anything about critical circle maps. The goal of this paper is to study the conjugacies of critical circle maps to a golden mean rotation and assess their regularity and asymptotic scaling properties. To motivate our subsequent analysis, we start with some preliminary visual explorations.

In Figure 2, we show two $\theta^{\mathrm{NC}}$ and one $\theta^{\mathrm{CC}}$. Obviously the $\theta^{\mathrm{NC}_{\mathrm{S}}}$ are less differentiable then the $\theta^{\mathrm{CC}}$; visually, $\theta^{\mathrm{CC}}$ is smoother than $C^{1}$.

In Figure 3, we show the conjugacies between a map of type N (resp., C, Q) and a Q map. Again, the conjugacy between two maps of the same type is evidently more differentiable than the ones between maps of different types. 


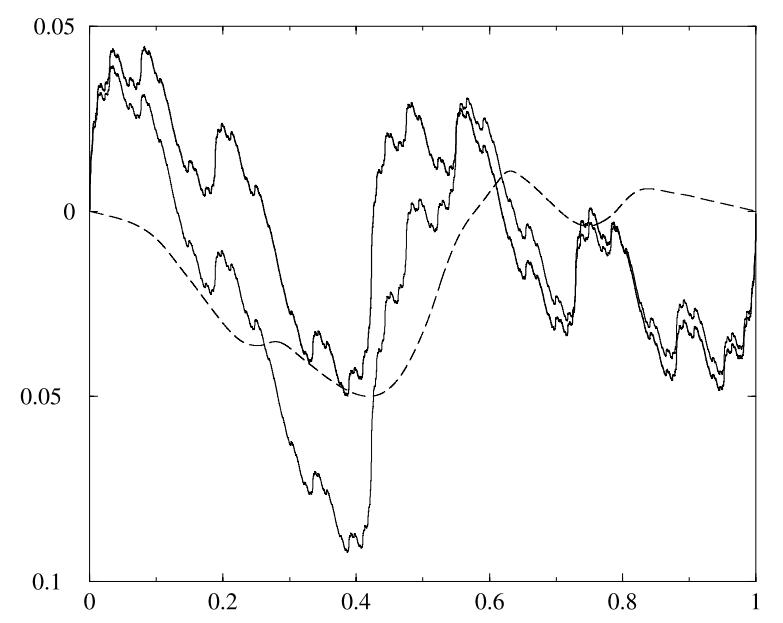

FIGURE 2. Conjugacies $\theta$ between: $f_{0.2}^{\mathrm{N}}$ and $f_{0.3}^{\mathrm{C}}$ (thin solid line), $f_{0.2}^{\mathrm{N}}$ and $f_{0.6}^{\mathrm{C}}$ (thick solid line), and $f_{0.6}^{\mathrm{C}}$ and $f_{0.3}^{\mathrm{C}}$ (dashed line).

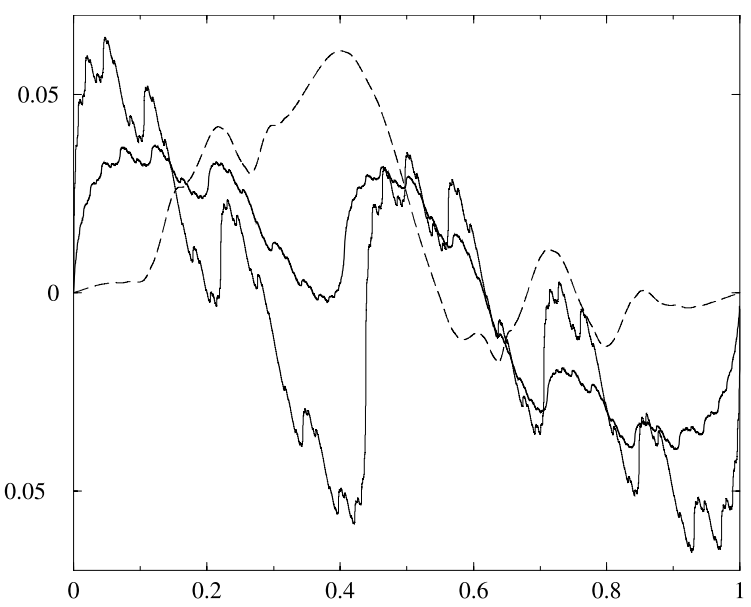

FIGURE 3. Conjugacies $\theta$ between: $f_{0.3}^{\mathrm{N}}$ and $f_{0.9}^{\mathrm{Q}}$ (thin solid line), $f_{0.6}^{\mathrm{C}}$ and $f_{0.9}^{\mathrm{Q}}$ (thick solid line), and $f_{0.6}^{\mathrm{Q}}$ and $f_{0.9}^{\mathrm{Q}}$ (dashed line).

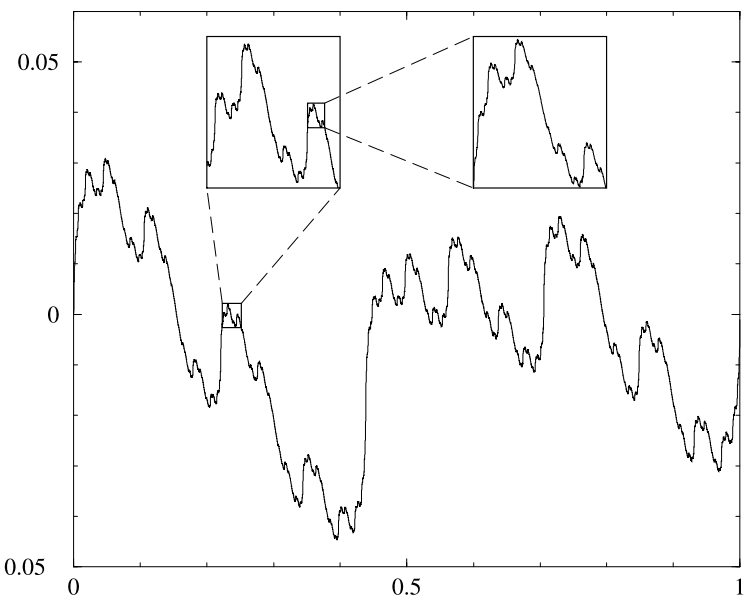

FIGURE 4. Zooming in the graph of the conjugacy between $f_{0.8}^{\mathrm{N}}$ and $f_{0.9}^{\mathrm{Q}}$.

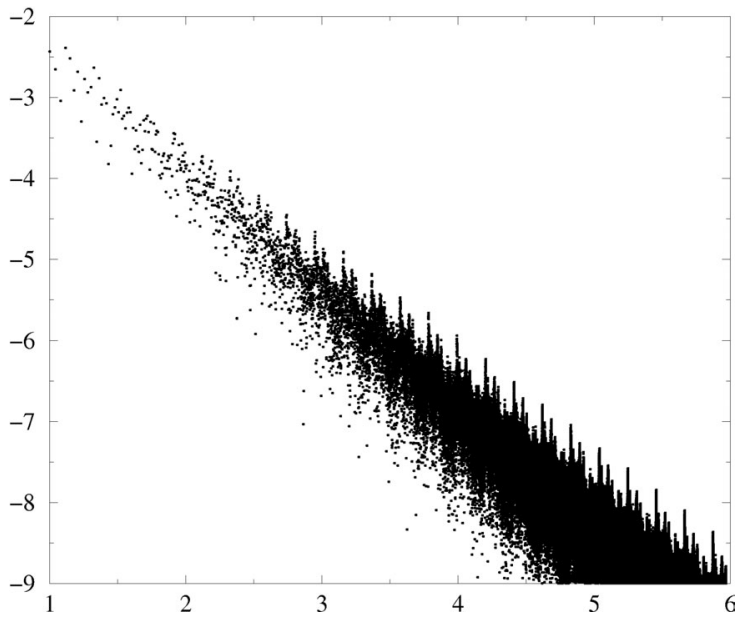

FIGURE 5. Plot of $\log _{10}\left|\hat{\theta}_{k}\right|$ vs. $\log _{10} k$ where $\theta$ is the conjugacy between $f_{0.2}^{\mathrm{N}}$ and $f_{0.6}^{\mathrm{C}}$.

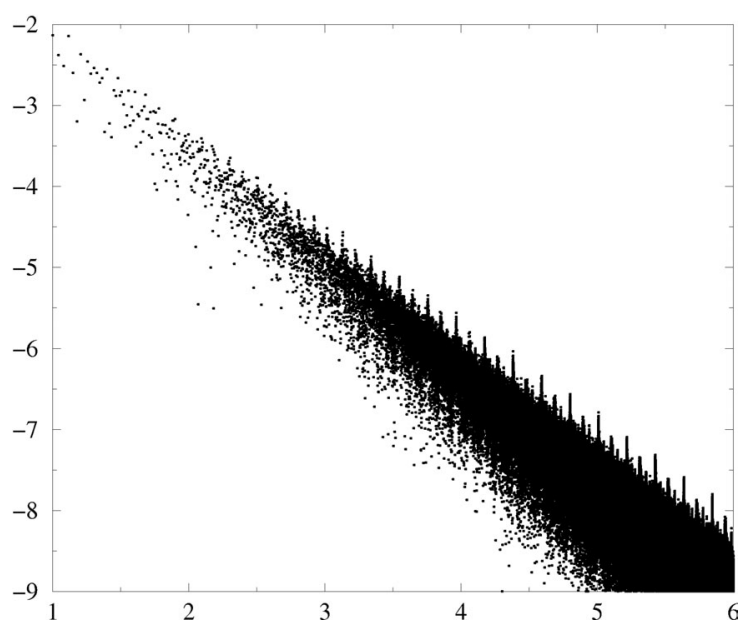

FIGURE 6. Plot of $\log _{10}\left|\hat{\theta}_{k}\right|$ vs. $\log _{10} k$ where $\theta$ is the conjugacy between $f_{0.3}^{\mathrm{N}}$ and $f_{0.9}^{\mathrm{Q}}$.

Another observation is the self-similar structure of the conjugacy between an $\mathrm{N}$ map and a critical ( $\mathrm{C}$ or $\mathrm{Q}$ ) map. To illustrate this, in Figure 4 we show magnified regions of the conjugacy between $f_{0.8}^{\mathrm{N}}$ and $f_{0.9}^{\mathrm{Q}}$. The selfsimilarity of the conjugacies between an $\mathrm{N}$ and a $\mathrm{C}$ map is one of the predictions of the theory of renormalization for C maps; we observed a self-similar structure in the case of the conjugacy between an $\mathrm{N}$ map and a $\mathrm{Q}$ map as well.

The self-similarity of the conjugacies of type $\theta^{\mathrm{NC}}$ and $\theta^{\mathrm{NQ}}$ can be seen distinctly from their Fourier spectra displayed in log-log form (Figures 5 and 6 ). The selfsimilarity manifests itself in the "periodicity" of the Fourier spectrum for large $|k|$. 


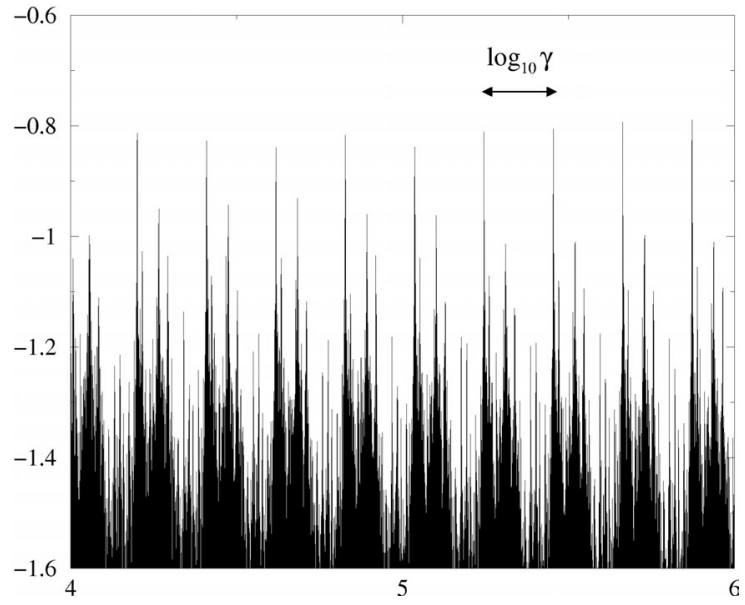

FIGURE 7. Plot of $\log _{10}\left(|k|^{1.29}\left|\hat{\theta}_{k}\right|\right)$ vs. $\log _{10}|k|$ where $\theta$ is the conjugacy betweeh $f_{0.2}^{\mathrm{N}}$ and $f_{1.0}^{\mathrm{C}}$.

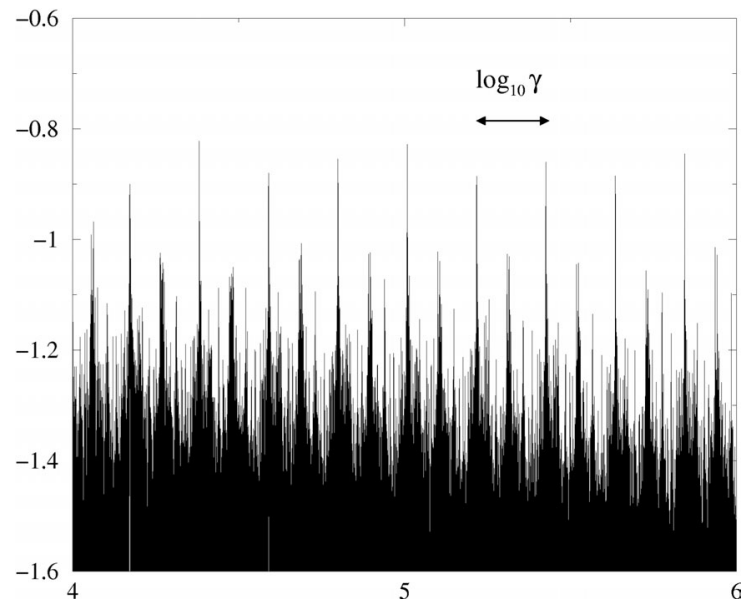

FIGURE 8. Plot of $\log _{10}\left(|k|^{1.19}\left|\hat{\theta}_{k}\right|\right)$ vs. $\log _{10}|k|$ where $\theta$ is the conjugacy between $f_{0.2}^{\mathrm{N}}$ and $f_{0.6}^{\mathrm{Q}}$.

This effect becomes even more prominent in the plot of $\log _{10}\left(|k|^{\lambda}\left|\hat{\theta}_{k}\right|\right)$ vs. $\log _{10}|k|$, as shown in Figure 7 (for $\theta^{\mathrm{NC}}, \lambda=1.29$ ) and Figure 8 (for $\theta^{\mathrm{NQ}}, \lambda=1.19$ ). In both cases, the width of the "periodic windows" is approximately equal to $\log _{10} \gamma$, as predicted by renormalization theory.

\section{METHODS FOR STUDYING REGULARITY}

In this section, we describe the function spaces studied and collect the theorems from harmonic analysis we used to compute the regularity of conjugacies.

\subsection{Hölder Spaces}

Let $C^{n}(\mathbb{T})(n \in \mathbb{N})$ stand for the space of $n$ times continuously differentiable functions on $\mathbb{T}$.
Definition 5.1. The Hölder spaces $\Lambda_{\alpha}(\mathbb{T})$ are defined as follows:

(i) For $\alpha \in(0,1)$ :

$$
\begin{gathered}
\|\theta\|_{\Lambda_{\alpha}(\mathbb{T})}:=\sup _{|y|>0} \frac{|\theta(x+y)-\theta(x)|}{|y|^{\alpha}}, \\
\Lambda_{\alpha}(\mathbb{T}):=\left\{\theta \in L^{\infty}(\mathbb{T}):\|\theta\|_{\Lambda_{\alpha}(\mathbb{T})}<\infty\right\} .
\end{gathered}
$$

(ii) For $\alpha=n+\alpha^{\prime}\left(n \in \mathbb{N}, \alpha^{\prime} \in(0,1)\right)$ :

$$
\Lambda_{\alpha}(\mathbb{T}):=\left\{\theta \in C^{n}(\mathbb{T}): \theta^{(n)} \in \Lambda_{\alpha^{\prime}}(\mathbb{T})\right\} .
$$

(iii) For $\alpha=1$ :

$$
\begin{aligned}
\|\theta\|_{\Lambda_{1}(\mathbb{T})} & :=\|\theta\|_{L^{\infty}(\mathbb{T})}+\sup _{|y|>0} \frac{|\theta(x+y)+\theta(x-y)-2 \theta(x)|}{|y|}, \\
\Lambda_{1}(\mathbb{T}) & :=\left\{\theta \in L^{\infty}(\mathbb{T}) \cap C^{0}(\mathbb{T}):\|\theta\|_{\Lambda_{1}(\mathbb{T})}<\infty\right\} .
\end{aligned}
$$

(iv) For $\alpha=n \in\{2,3,4, \ldots\}$ :

$$
\Lambda_{n}(\mathbb{T}):=\left\{\theta \in L^{\infty}(\mathbb{T}) \cap C^{n-1}(\mathbb{T}): \theta^{(n-1)} \in \Lambda_{1}(\mathbb{T})\right\} .
$$

\section{Remark 5.2.}

1. $C^{1}(\mathbb{T}) \subset \operatorname{Lip}(\mathbb{T}) \subset \Lambda_{1}(\mathbb{T})$ and $C^{n}(\mathbb{T}) \subset \Lambda_{n}(\mathbb{T})(n \geq$ 2); all these inclusions are strict.

2. Every $\theta \in \Lambda_{\alpha}(\mathbb{T})(0<\alpha)$ may be modified on a set of measure zero so that it becomes continuous [Stein 70, Sec. V.4.1].

The spaces in these scales have several characterizations some of which lead to algorithms that can be used to assess the regularity of functions numerically. Some of these characterizations will de discussed in Sections 5.25.4. The numerical implementation of these methods will be discussed in Section 6 .

\subsection{Finite Difference Method}

We now look at the characterization of Hölder spaces by means of finite differences (FD) [Krantz 83]. Let $\mathcal{D}_{y}^{n}$ be the finite difference operator:

$$
\left(\mathcal{D}_{y}^{n} \theta\right)(x):=\sum_{j=0}^{n}(-1)^{j}\left(\begin{array}{l}
n \\
j
\end{array}\right) \theta(x+(n-2 j) y) .
$$

Theorem 5.3. (FD.) Let $\theta \in L^{\infty}(\mathbb{T}) \cap C^{0}(\mathbb{T})$ and $0<\alpha<$ $n \in \mathbb{Z}$. Then $\theta \in \Lambda_{\alpha}(\mathbb{T})$ if and only if $\exists C>0$ such that $\forall y \in \mathbb{T}$

$$
\left\|\mathcal{D}_{y}^{n} \theta\right\|_{L^{\infty}(\mathbb{T})} \leq C|y|^{\alpha}, \quad \text { for all } y \in \mathbb{T} .
$$


The FD method is simple and convenient to use if one can compute the values of the function in points that are arbitrarily close and equally spaced. As mentioned before, this requires interpolation between the iterates $f^{n}(0)$.

\subsection{Fourier Methods-Littlewood-Paley Theorem}

The trigonometric system $\left\{e^{2 \pi i k x}\right\}_{k \in \mathbb{Z}}$ is an orthonormal basis of $L^{2}(\mathbb{T}, d x)$; hence, according to Plancherel's theorem, a function

$$
\theta(x)=\sum_{k \in \mathbb{Z}} \hat{\theta}_{k} e^{2 \pi i k x}
$$

belongs to $L^{2}(\mathbb{T})$ if and only if

$$
\sum_{k \in \mathbb{Z}}\left|\hat{\theta}_{k}\right|^{2}<\infty
$$

The main result of the Littlewood-Paley theory is that similar characterization of $L^{p}(\mathbb{T})(1<p<\infty)$ can be obtained by grouping the terms of the Fourier series in dyadic blocks. Define the decomposition

$$
\theta=\sum_{M=1}^{\infty} \mathcal{L}_{M} \theta
$$

of $\theta \in L^{1}(\mathbb{T})$ in dyadic partial sums

$$
\left(\mathcal{L}_{M} \theta\right)(x):=\sum_{A^{M-1} \leq|k|<A^{M}} \hat{\theta}_{k} e^{2 \pi i k x}
$$

$(M \in \mathbb{N}), \mathcal{L}_{0} \theta:=\hat{\theta}_{0}$, and $A>1$.

Remark 5.4. Usually, $A$ is taken to be 2 , since the precise value does not make any difference for the mathematical treatment. In the numerical applications, we will find it convenient to use some values of $A$ other than 2. Nevertheless, we have not introduced $A$ in the notation, since it will be clear from the context, and we follow the standard practice of calling the decomposition "dyadic."

The dyadic blocks can be written as

$$
\mathcal{L}_{M} \theta=\left(\phi_{A^{M}}-\phi_{A^{M-1}}\right) * \theta,
$$

where the function

$$
\phi_{N}(x):=\sum_{|k|<N} e^{2 \pi i k x}
$$

plays a role of a "low-pass filter," or, in the terminology of physicists, introduces an "ultraviolet" cutoff.
To formulate the celebrated Littlewood-Paley (LP) theorem, we introduce the Littlewood-Paley $d$-function,

$$
d(\theta)(x):=\left(\sum_{M=0}^{\infty}\left|\mathcal{L}_{M} \theta(x)\right|^{2}\right)^{1 / 2},
$$

and its "continuous" analog, the $G$-function,

$$
G(\theta)(x):=\left(\int_{0}^{1}(1-s)\left|\left(\frac{d P_{s}}{d s} * \theta\right)(x)\right|^{2} d s\right)^{1 / 2},
$$

where

$$
\begin{aligned}
P_{s}(x) & =\sum_{k \in \mathbb{Z}} s^{|k|} e^{2 \pi i k x} \\
& =\frac{1-s^{2}}{1-2 s \cos 2 \pi x+s^{2}}, \quad s \in[0,1)
\end{aligned}
$$

is the periodic Poisson kernel. Note that if $\Delta$ is the Laplacian, then

$$
\begin{aligned}
P_{\exp (-2 \pi t)} * \theta(x) & =e^{-t \sqrt{-\Delta}} \theta(x) \\
& =\sum_{k \in \mathbb{Z}} \hat{\theta}_{k} e^{-2 \pi t|k|} e^{2 \pi i k x} .
\end{aligned}
$$

Heuristically, it seems clear that the partial sums, $\phi_{n} *$ $\theta$, behave like the Abel means, $P_{1-\frac{1}{n}} * \theta$. In fact, one can prove that the $L^{p}(\mathbb{T})$ norms of $d(\theta)$ and $G(\theta)$ are equivalent for $1<p<\infty$ if $\hat{\theta}_{0}=0$.

Remark 5.5. The Poisson kernel can also be considered as defined on the real line. In that case, it can be given by the formula $P_{t}=e^{-t \sqrt{-\Delta}}$ or as the convolution with the kernel $P_{t}(x)=\pi^{-1 / 2} t /\left(x^{2}+t^{2}\right)$.

We can consider a periodic function of period 1 on the real line as a function on the circle. When we apply the real Poisson kernel to a periodic function of period 1, it also produces a periodic function of period 1 .

It is well-known and not difficult to check (Poisson summation formula) that applying the real Poisson kernel to a periodic function of period one defined on $\mathbb{R}$, and considering the function as defined on the circle and applying the periodic Poisson kernel (5-5) are the same.

Remark 5.6. On the real line, it makes sense to define scaling transformations and to investigate how the Poisson kernel behaves under scalings. It is very easy to check that, for every $\lambda>0$, the Poisson kernel on $\mathbb{R}$ satisfies

$$
P_{\lambda t}(\lambda x)=\lambda^{-1} P_{t}(x) .
$$

On the circle, we cannot speak about scaling, therefore the relation (5-6) does not, strictly speaking, make 
sense for the Poisson kernel on the circle when $\lambda$ is not an integer. Nevertheless, for small scales, the circle can be identified with the real line so that the scalings of the periodic Poisson kernel can be used when examining asymptotic features in small scales.

Theorem 5.7. (Littlewood-Paley.) If $\theta \in L^{p}(\mathbb{T}), 1<p<$ $\infty$, then there exist positive constants $A_{p}$ and $B_{p}$ such that

$$
A_{p}\|\theta\|_{L^{p}(\mathbb{T})} \leq\|d(\theta)\|_{L^{p}(\mathbb{T})} \leq B_{p}\|\theta\|_{L^{p}(\mathbb{T})} .
$$

Analogous inequalities hold for $G(\theta)$ in place of $d(\theta)$.

Theorem 5.7 has many important implications. In particular, it gives useful characterizations of Sobolev, Hölder, Hardy, Besov spaces - see [Stein 70, Ch. 5], [Hernández and Weiss 96, Ch. 6], [Meyer 90, Ch. 6], and [Frazier et al. 91].

In our numerical explorations, we use methods based on the following two corollaries of Theorem 5.7, which we will call "discrete" (DLP) and "continuous" (CLP) versions of the Littlewood-Paley theorem.

Theorem 5.8. (DLP.) The function $\theta$ (5-2) is of class $\Lambda_{\alpha}(\mathbb{T})\left(\alpha \in \mathbb{R}_{+}\right)$if and only if there exists a $C>0$ such that for any $M \in \mathbb{N}$ [Krantz 83, Theorem 5.9]

$$
\left\|\mathcal{L}_{M} \theta\right\|_{L^{\infty}(\mathbb{T})} \leq C A^{-\alpha M} .
$$

Theorem 5.9. (CLP.) The function $\theta$ (5-2) is of class $\Lambda_{\alpha}(\mathbb{T})\left(\alpha \in \mathbb{R}_{+}\right)$if and only if for each $\eta \geq 0$ there exists $a C>0$ such that for any $t>0$ [Stein 70, Ch. 5 , Lemma $5]$

$$
\left\|\left(\frac{\partial}{\partial t}\right)^{\eta} e^{-t \sqrt{-\Delta}} \theta\right\|_{L^{\infty}(\mathbb{T})} \leq C t^{\alpha-\eta} .
$$

\subsection{Wavelet Methods}

The guiding idea of wavelet theory is to decompose functions systematically into functions that have definite scales decreasing geometrically. This is, of course, related to the decompositions used in Littlewood-Paley (cf. (5$3)$ ).

Expansions in wavelet bases are very well-suited to studying the local properties of functions because of their localization in space. Wavelet methods are especially appropriate for analyzing self-similar functions like some of the conjugacies between circle maps studied in this paper. Below we introduce the notations and collect the basic theoretical results about regularity of functions expanded in wavelet bases. For more details, see [Meyer 90], [Daubechies 92], [Mallat 98], [Hernández and Weiss 96], [Härdle et al. 98], and [Louis et al. 97].

Let $L^{2}(\mathbb{T})_{2^{L}}$ be the "discrete" version of the space of square integrable circle maps, i.e., the $2^{L}$-dimensional space of the circle maps defined on the grid $x_{\ell}=2^{-L} \ell$, $\ell=0,1, \ldots, 2^{L}-1$. We use the following multiresolution analysis of $L^{2}(\mathbb{T})_{2^{L}}$ :

$$
V_{0} \subset V_{1} \subset \cdots \subset V_{L-1} \subset V_{L}=L^{2}(\mathbb{T})_{2^{L}} .
$$

Let $W_{j}$ be the orthogonal complement of $V_{j}$ in $V_{j+1}$, so that

$$
\begin{gathered}
L^{2}(\mathbb{T})_{2^{L}}=V_{0} \oplus\left(\bigoplus_{j=0}^{L-1} W_{j}\right) ; \\
\operatorname{dim} V_{j}=\operatorname{dim} W_{j}=2^{j} .
\end{gathered}
$$

The space $W_{j}$ is spanned by $\left\{\psi_{j k}\right\}_{k=0}^{2^{j}-1}$, where

$$
\psi_{j k}(x)=2^{j / 2} \psi\left(2^{j} x-k\right)
$$

and $\psi$ is the "mother wavelet." Let $\theta_{2^{L}}:=\left\{\theta\left(x_{\ell}\right)\right\}_{\ell=0}^{2^{L}-1} \in$ $L^{2}(\mathbb{T})_{2^{L}}$ be the discrete representation of the function $\theta$, and

$$
\Pi_{J}: L^{2}(\mathbb{T})_{2^{L}} \rightarrow V_{J}: \theta_{2^{L}} \mapsto \sum_{j=0}^{J} \sum_{k=0}^{2^{j}-1}\left\langle\theta, \psi_{j k}\right\rangle \psi_{j k}
$$

be the projections onto $V_{J}, J=0,1, \ldots, L$.

The Littlewood-Paley theorem can be generalized to bases other than the trigonometric one by observing that the proofs do not use the explicit form of $\phi_{N}(5-4)$ and $P_{s}(5-5)$, but only some of their properties, so that the results are valid for larger function classes. In particular, the following theorem holds:

Theorem 5.10. If $\psi \in \Lambda_{\alpha}(\mathbb{T})$, then the function $\theta$ is of class $\Lambda_{\alpha}(\mathbb{T})$ if and only if there exists a $C>0$ such that for any $j \in \mathbb{N}$ [Hernández and Weiss 96, Theorem 7.16]

$$
\sup _{0 \leq k \leq 2^{j}-1}\left|\left\langle\theta, \psi_{j k}\right\rangle\right| \leq C 2^{-j\left(\alpha+\frac{1}{2}\right)} .
$$

Another formulation which is useful for numerical computations is

Theorem 5.11. If $\psi \in \Lambda_{\alpha}(\mathbb{T})$, then the function $\theta$ is of class $\Lambda_{\alpha}(\mathbb{T})$ if and only if there exists a $C>0$ such that 
for any $j \in \mathbb{N}$,

$$
\left\|\theta-\Pi_{j} \theta\right\|_{L^{\infty}(\mathbb{T})} \leq C 2^{-j \alpha} .
$$

For more subtle results on applications of wavelets to studies of local regularity of functions, see [Jaffard and Meyer 96], [Holschneider and Tchamitchian 91], [Jaffard 97], and [Meyer 98]. We will not explore local regularity here, even if our numerical methods are related to the results in [Jaffard 97].

\section{NUMERICAL IMPLEMENTATION}

\subsection{General Remarks}

The characterizations mentioned above involve inequalities that have to be satisfied for an infinite number of integers. Obviously, the numerical calculation can only compute the Fourier and wavelet transform up to a finite order. It is conceivable that the behavior of the functions is different for high Fourier modes than for the values that can be explored.

In spite of the above solipsistic argument, there are good reasons (a renormalization group description) that strongly suggest that the functions we are studying are asymptotically self-similar, so that the the study of a finite number of scales accurately predicts the behavior at all scales. Indeed, we find empirically that the upper bounds giving the regularity become approximately identities. We see that, after a very short transient, the upper bounds become identities up to a small periodic error whose interpretation we will discuss in Section 6.5.

Because of this empirical observation and the renormalization group description, we believe that it is reasonable to extrapolate from the observed values and conclude that the upper bounds giving regularity are saturated to all scales.

Another issue that one has to discuss in numerical implementations is the effect of the round off and discretization error. This analysis is very similar to the standard considerations of numerical analysis.

Finding numerically the regularity of functions that are very smooth is difficult because their Fourier/wavelet coefficients decrease faster. That is why we were not able to assess the precise values of the smoothness of the conjugacies of type $\theta^{\mathrm{CC}}$ and $\theta^{\mathrm{QQ}}$, whose smoothness is more than one.

In these two cases, as well as for all conjugacies between $f$ and $g$ for $f$ being critical (C or Q), an important issue is the presence of big gaps between the iterates $f^{n}(0)$ (see Section 4.3). This is because we perform a Fast Fourier Transform (FFT) or Discrete Wavelet Transform (DWT) not on the exact values of $\theta$ at the points $x_{\ell}$ (4-9), but on the values of the interpolating cubic polynomials at these points, which significantly deteriorates the precision of the spectra.

For the FFT, we used the routines four1 and realft from [Press et al. 92] (for long double precision). For the DWT, we used the freely available $\mathrm{C}$ routines documented in detail in the book [Wickerhauser 94]. For the graphing and some of the data analysis, we used the plotting tool ACE/gr.

Numerically, the most important restriction on the number of Fourier or wavelet coefficients computed was not the speed, but the memory usage (in some of the cases, about $200 \mathrm{Mb}$ ).

\subsection{Calibration of the Methods}

To assess the validity of the numerical methods that have been employed, we have taken an empirical approach, testing them on functions whose regularity is known. One particularly good class of functions for calibration is the Weierstrass functions,

$$
w_{a, b}(x)=\sum_{k=1}^{\infty} a^{k} \sin \left(2 \pi b^{k} x\right),
$$

where $a<1, b \in \mathbb{N}$. As it is well known, $w_{a, b} \in \Lambda_{-\log _{b} a}$, and for any $\delta>0, w_{a, b} \notin \Lambda_{-\log _{b} a+\delta \text {. }}$

To calibrate our numerical methods, we have generated the $w_{a, b}$ functions at points obtained by iterating the diffeomorphisms we are studying. Then, we obtained the regularity applying the methods outlined above. This procedure gave us an idea of the severity of the problem of the lack of equidistribution of the iterates. The use of the Weierstrass function to calibrate the methods seems appropriate because the working hypothesis (A3) asserts that the functions we are studying are very similar to the functions $(6-1)$. Hence, one can hope that the problems of interpolation and lack of distribution can be assessed by testing the methods on $(6-1)$.

\subsection{Finite Differences Method}

We applied Theorem 5.3 for $y=2^{-j}$, in which case (5-1) yields

$$
\log _{2}\left\|\mathcal{D}_{2^{-j}}^{n} \theta\right\|_{L^{\infty}(\mathbb{T})} \leq \text { const }-\alpha j
$$

(naturally, one can consider the case of arbitrary $y$ ). As examples of the results obtained by applying this method, we show in Figure 9 the plot of $\log _{2}\left\|\mathcal{D}_{2^{-j}}^{1} \theta\right\|_{L^{\infty}(\mathbb{T})}$ as a function of $j$ for four conjugacies of type $\mathrm{NC}$ (x's) and 


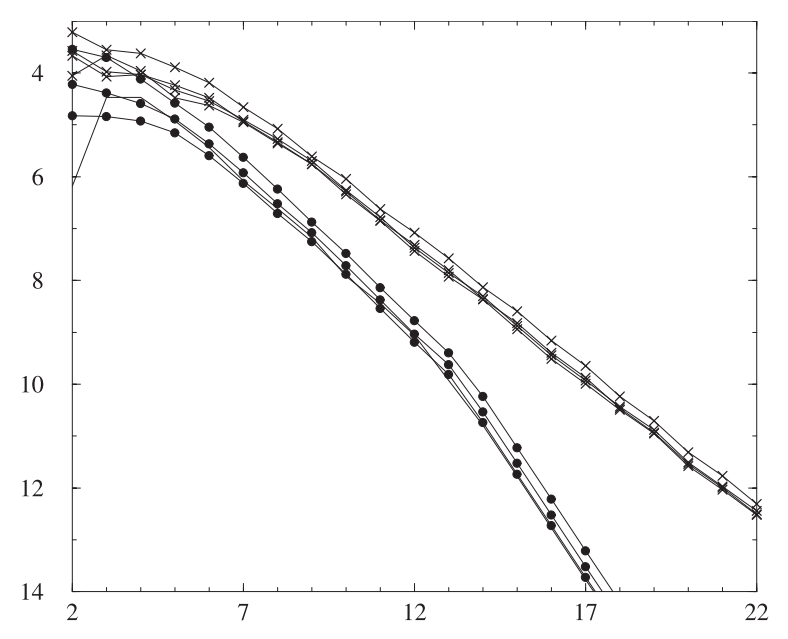

FIGURE 9. Plot of $\log _{2}\left\|\mathcal{D}_{2^{-j}}^{1} \theta\right\|_{L^{\infty}(\mathbb{T})}$ vs. $j$ for four $\theta^{\mathrm{NC}}$ 's (x's) and four $\theta^{\mathrm{CQ}}$ 's (circles).

four ones of type CQ (circles); to calculate $\theta^{\mathrm{NC}}$, we used $10^{7}$ iterates and $2^{22}$ interpolated values, while for $\theta^{\mathrm{CQ}}$, these numbers were $2 \times 10^{6}$ and $2^{22}$, respectively.

In the favorable case (NC), we see that the numerical results correspond to parallel straight lines that cover the whole range plotted. On the other hand, in the unfavorable case (CQ), the numerical results present two straight lines joined by a break.

This can be clearly explained because the graph presented for the NC case includes computations in which many of the points in the finite difference operator are included in the gaps. Hence, the finite difference operator is observing the regularity of the interpolating spline.

In the NC case, the gaps between the iterates did not exceed $1.5 \times 10^{-7}$. In the $\mathrm{CQ}$ case, the maximum gap was about $2 \times 10^{-4} \approx 2^{-12}$, which corresponds quite exactly to the position of the break in the graph. When we restrict the differences to regions larger than the gaps, the method produces results consistent with the other methods.

\subsection{DLP Method}

Theorem 5.8 implies that

$$
\log _{A}\left\|\mathcal{L}_{M} \theta\right\|_{L^{\infty}(\mathbb{T})} \leq \text { const }-\alpha M
$$

i.e., the Hölder exponent of $\theta$ is the negative of the slope of the graph of $\log _{A}\left\|\mathcal{L}_{M} \theta\right\|_{L^{\infty}(\mathbb{T})}$ vs. $M$.

Graphs of this type for some classes of conjugacies are shown in Figure 10. Each case is represented by two conjugacies, the first one depicted by a big empty shape, and the second one by a small full shape: $\left(f_{0.3}^{\mathrm{N}}, f_{0.6}^{\mathrm{C}}\right)$ and $\left(f_{0.3}^{\mathrm{N}}, f_{0.7}^{\mathrm{C}}\right)$-circles; $\left(f_{0.5}^{\mathrm{N}}, f_{0.6}^{\mathrm{Q}}\right)$ and $\left(f_{0.5}^{\mathrm{N}}, f_{0.9}^{\mathrm{Q}}\right)$-squares;

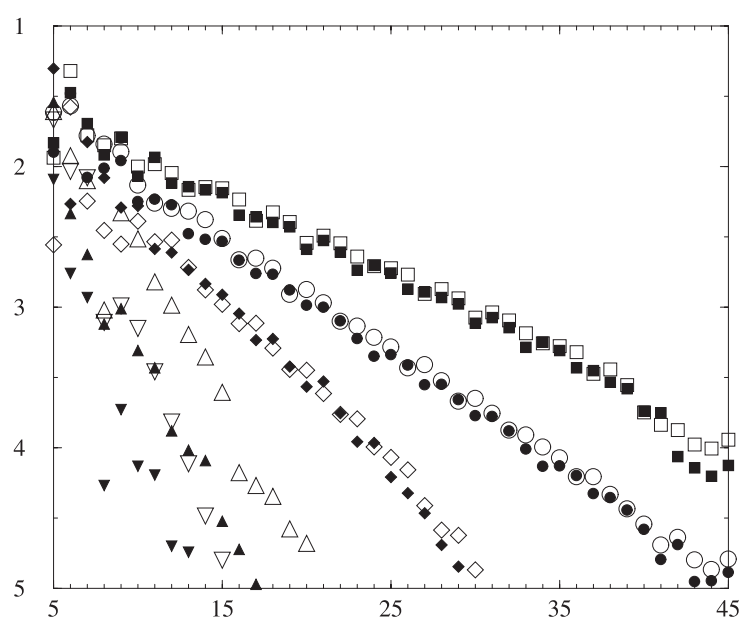

FIGURE 10. Plot of $\log _{10}\left\|\mathcal{L}_{M} \theta\right\|_{L^{\infty}(\mathbb{T})}$ vs. $M$ (for $A=1.4$ ) for pairs of conjugacies of five different types.

$\left(f_{0.6}^{\mathrm{C}}, f_{0.6}^{\mathrm{Q}}\right)$ and $\left(f_{0.3}^{\mathrm{C}}, f_{0.9}^{\mathrm{Q}}\right)$ - diamonds; $\left(f_{0.6}^{\mathrm{C}}, f_{0.3}^{\mathrm{C}}\right)$ and $\left(f_{0.7}^{\mathrm{C}}, f_{0.6}^{\mathrm{C}}\right)$-triangles down; $\left(f_{0.6}^{\mathrm{Q}}, f_{0.9}^{\mathrm{Q}}\right)$ and $\left(f_{0.9}^{\mathrm{Q}}, f_{1.2}^{\mathrm{Q}}\right)$ triangles up. Clearly, the smoothness of the conjugacies of different classes is different, but this graph does not allow us to find the smoothness of the conjugacies precisely (and for $\theta^{\mathrm{CC}}$ and $\theta^{\mathrm{QQ}}$, the results are very poor). The reasons for this are as follows:

First, each point on this graph is computed by using not all Fourier coefficients of $\theta$, but rather only a dyadic block of them, so for small $M$, the points on the graph are based on a small number of Fourier coefficients. For large $M$, the points are based on larger number of Fourier coefficients, but these coefficients are affected by the numerical noise. Also, the number of points in the figure is of order $\log _{A}$ of the number of Fourier coefficients found, i.e., it is significantly smaller than the number of coefficients. In our explorations we used values of $A$ around 1.5.

\subsection{CLP Method}

From a numerical point of view, the CLP method (based on Theorem 5.9) is much better than DLP. First of all, we can calculate $\left\|\frac{\partial^{\eta}}{\partial t^{\eta}} e^{-t \sqrt{-\Delta}} f\right\|_{L^{\infty}(\mathbb{T})}$ for as many values of $t$ as we wish. Furthermore, for each value of $t$, the value of this norm is based on the values of all known Fourier coefficients of $f$. Finally, one can perform calculations for different values of $\eta$ and check whether they yield the same value of $\alpha$-this is a very good test of the reliability of the numerical results.

To illustrate how well this method works, Figure 11 shows plots of $\log _{10}\left\|\frac{\partial^{2}}{\partial t^{2}} e^{-t \sqrt{-\Delta}} w_{0.57,3}\right\|_{L^{\infty}(\mathbb{T})}$ vs. $\log _{10} t$ for $2^{22}$ (circles), $2^{13}(\mathrm{x})$, and $2^{10}$ (pluses) Fourier components based on the values of $w_{0.57,3}$ at the points 


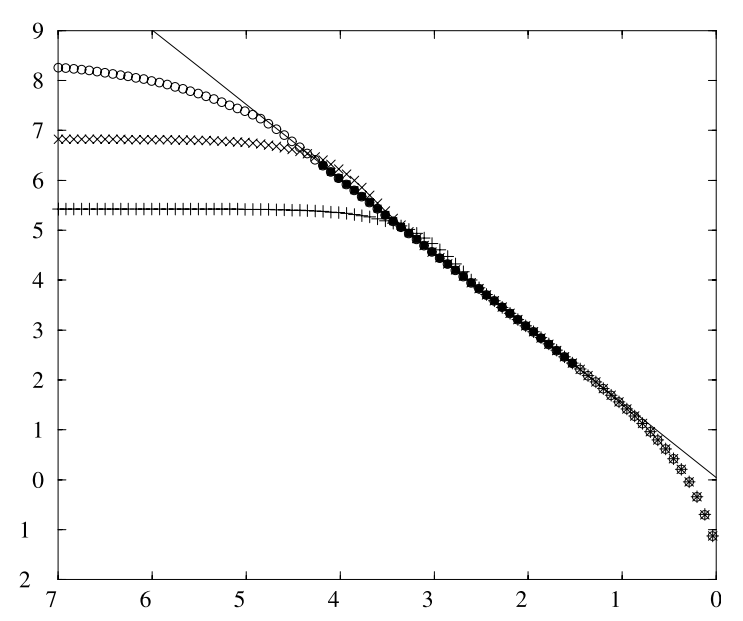

FIGURE 11. Plot of $\log _{10}\left\|\frac{\partial^{2}}{\partial t^{2}} e^{-t \sqrt{-\Delta}} w_{0.57,3}\right\|_{L^{\infty}(\mathbb{T})}$ vs. $\log _{10} t$.

$\left(f_{0.5}^{\mathrm{N}}\right)^{n}(0)$ for $n=0, \ldots, 2^{21}-1$. Evidently, the position of the plateau for small $t$ depends on the number of Fourier coefficients used in the computation. Theoretically, the regularity of $w_{0.57,3}$ is $-\log _{3} 0.57=0.5117 \ldots$ The slope of the straight line that best fits the full circles in the figure is -1.4908 , so the numerically found regularity according to $(5-8)$ is $2-1.4908=0.5092$ - a value that differs from the exact one by only 0.002 .

Figure 12 shows graphs of $\log _{10}\left\|\frac{\partial^{\eta}}{\partial t^{\eta}} e^{-t \sqrt{-\Delta}} \theta\right\|_{L^{\infty}(\mathbb{T})}$ vs. $\log _{10} t$ for $\eta=1,2,3 ; \theta$ is the conjugacy between $f_{0.2}^{\mathrm{N}}$ and $f_{0.6}^{\mathrm{C}}$. The results of the linear regression of these data are presented in Table 2. The uncertainties are just the the standard errors of the regression.

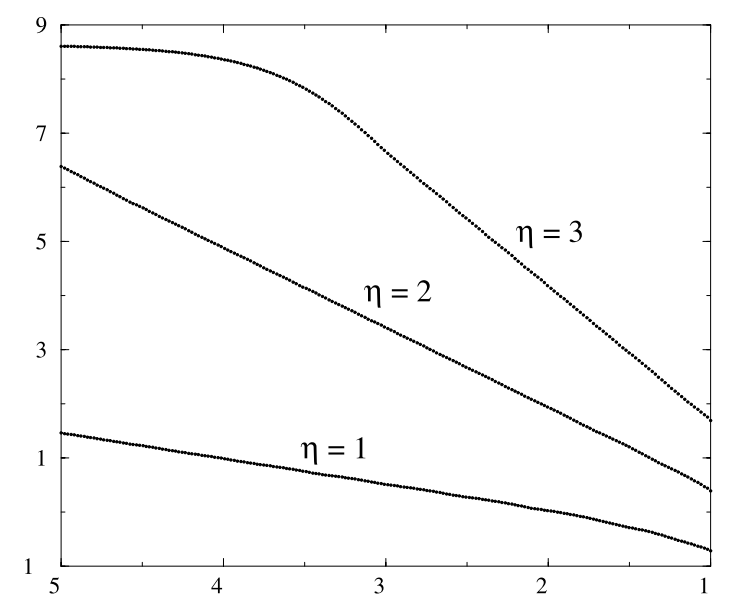

FIGURE 12. Plot of $\log _{10}\left\|\frac{\partial^{\eta}}{\partial t^{\eta}} e^{-t \sqrt{-\Delta}} \theta\right\|_{L^{\infty}(\mathbb{T})}$ vs. $\log _{10} t$ for $\eta=2$ and $\eta=3$ of all 12 conjugacies between an $\mathrm{N}$ and a $\mathrm{C}$ map for four $\mathrm{N}$ and three $\mathrm{C}$ maps with different parameter values. Each line connects 146 points; to obtain each point, we have used $10^{6}$ iterates and $2^{21} \approx 10^{6}$ spline points.

\begin{tabular}{|c|c|c|}
\hline$\eta$ & Range of $\log _{10} t$ & Regularity \\
\hline 1 & {$[-5.0,-4.0]$} & $0.5247 \pm 0.0009$ \\
\hline 2 & {$[-3.5,-2.5]$} & $0.5253 \pm 0.0012$ \\
\hline 3 & {$[-3.0,-1.5]$} & $0.5244 \pm 0.0008$ \\
\hline
\end{tabular}

TABLE 2. Regularity of the conjugacy between $f_{0.2}^{\mathrm{N}}$ and $f_{0.6}^{\mathrm{C}}$, found by linear regression of the data in Figure 12.

In Figure 13, we show $\log _{10}\left\|\frac{\partial^{\eta}}{\partial t^{\eta}} e^{-t \sqrt{-\Delta}} \theta\right\|_{L^{\infty}(\mathbb{T})}$ vs. $\log _{10} t$ for $\eta=1,2$ for all the 16 conjugacies between the four $\mathrm{N}$ and four $\mathrm{C}$ maps we considered. We call attention to the fact that the lines are not only parallel, but they are also very close.

The CLP method can be used also to test some features of the expansion (3-1). Since (3-1) is supposed to hold only in the asymptotic limit of very small scales, we can use Remark 5.6 and the scalings (5-6). Note that, taking the convolution of $(3-1)$ with the Poisson kernel and using (5-6), we obtain in the notation of Section 3:

$$
\begin{gathered}
P_{t} *\left[\sum_{n} \lambda_{1}^{n}\left(H_{1} \circ \alpha^{n}\right)(x)+\lambda_{2}^{n}\left(H_{2} \circ \alpha^{n}\right)(x)+\cdots\right] \\
=\sum_{n}\left[\begin{array}{c}
\lambda_{1}^{n}\left[P_{\alpha^{n} t} * H_{1}\right]\left(\alpha^{n} x\right) \\
\left.+\lambda_{2}^{n}\left[P_{\alpha^{n} t} * H_{2}\right]\left(\alpha^{n} x\right)+\cdots\right]
\end{array}\right.
\end{gathered}
$$

If we take suprema in $x$ and then logarithms, the structure of the main term for the resulting function considered as a function of $\log t$ is a sum of a linear function and a function that is periodic. The slope of the linear function is, of course, according to Theorem 5.9, the degree of differentiability, but if we subtract the linear part, we should see the periodicity.

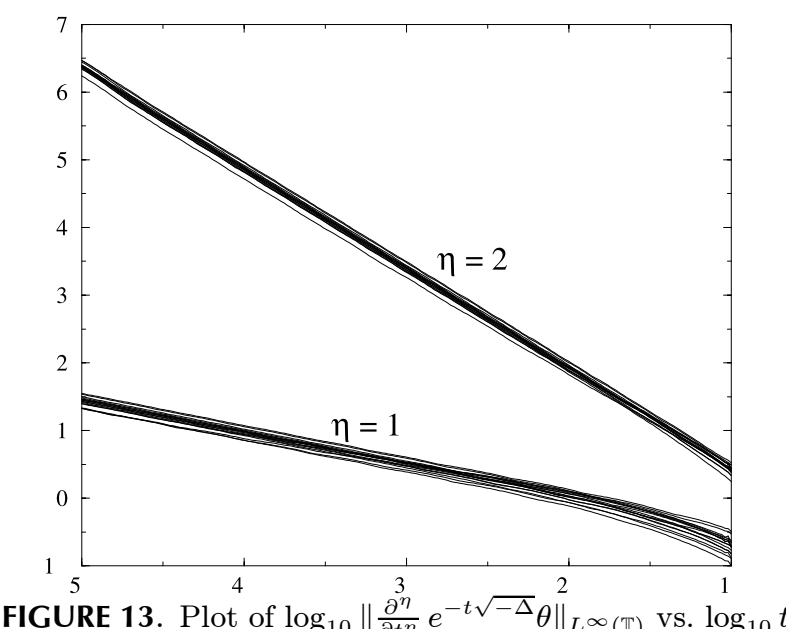

FIGURE 13. Plot of $\log _{10}\left\|\frac{\partial^{\eta}}{\partial t^{\eta}} e^{-t \sqrt{-\Delta}} \theta\right\|_{L^{\infty}(\mathbb{T})}$ vs. $\log _{10} t$ for $\eta=1,2$ for 16 conjugacies of type NC. 


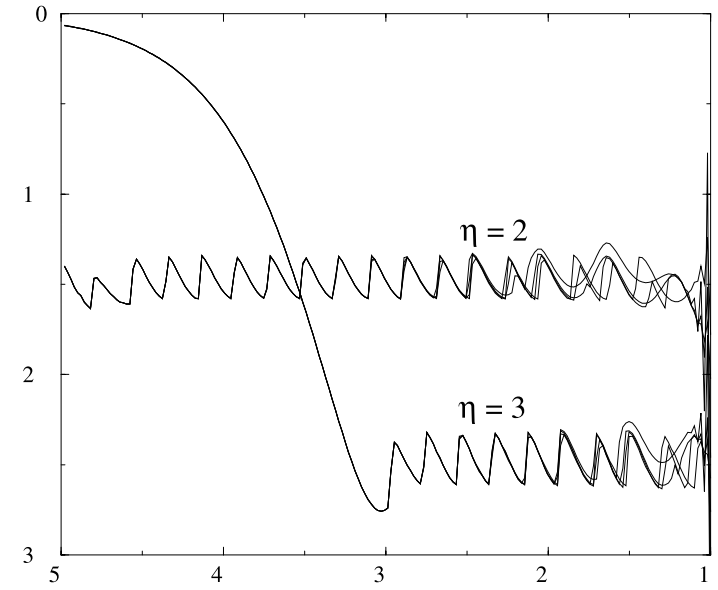

FIGURE 14. Plot of the first differences of the graph of $\log _{10}\left\|\frac{\partial^{\eta}}{\partial t^{\eta}} e^{-t \sqrt{-\Delta}} \theta\right\|_{L^{\infty}(\mathbb{T})}$ (in arbitrary units) vs. $\log _{10} t$ for $\eta=2$ and $\eta=3$ for four $\theta$ of type NC.

This exploration of the first differences is undertaken in Figure 14, where we plot the first differences of the graph of $\log _{10}\left\|\frac{\partial^{\eta}}{\partial t^{\eta}} e^{-t \sqrt{-\Delta}} \theta\right\|_{L^{\infty}(\mathbb{T})}$ as a function of $\log _{10} t$ (for $\eta=2,3$ ) at equally spaced points.

Note that taking first differences turns a linear function into a constant, and a periodic function into a periodic function. Higher order differences eliminate the linear function and receive contributions of the periodic part.

In Figure 15, we show the same plot as above for four conjugacies of type NQ and the first and second differences of the plot. We call attention to the fact that the periodic corrections we plot quickly become independent of the functions we start with, which corresponds to the fact that the function $H_{1}$ is universal. This is particu-

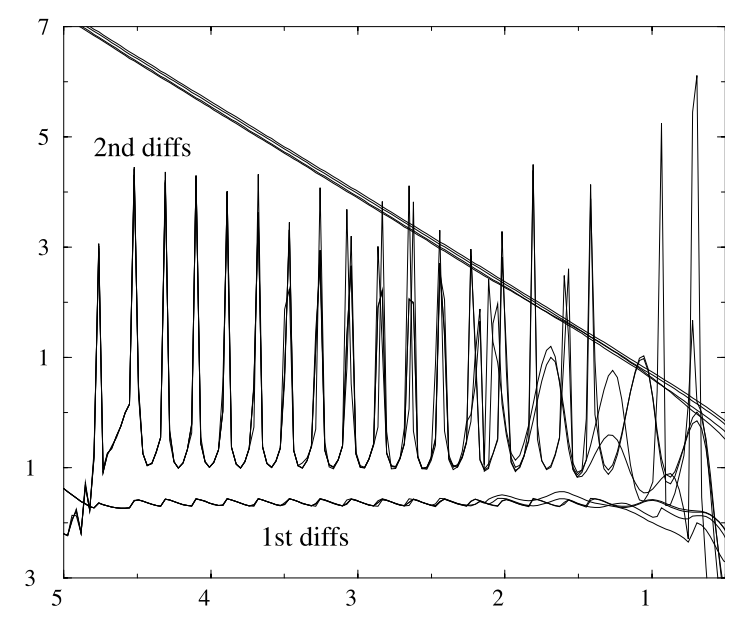

FIGURE 15. Plot of $\log _{10}\left\|\frac{\partial^{2}}{\partial t^{2}} e^{-t \sqrt{-\Delta}} \theta\right\|_{L^{\infty}(\mathbb{T})}$ vs. $\log _{10} t$ for four $\theta$ of type NC and the first and second differences. larly remarkable for the case of second differences since they are very susceptible to numerical errors. Hence, this gives us confidence on the reliability of the methods we have used.

We note that the computation of first differences is one of the data analysis features included in ACE/gr, so it is quite feasible to carry out these explorations in an interactive way for a variety of functions.

\subsection{Wavelet Coefficient Decay}

Theorem 5.10 can be used to assess the regularity of the functions we study by examining the decay of the coefficients of the wavelet transform. Nevertheless, we do not think that for our functions it is necessary to appeal to Theorem 5.10.

Note that the working hypothesis (A4) gives a representation of the function. It is not difficult to show that, for functions of the form (3-1) in the working hypothesis (A4), the degree of regularity is a simple ratio between the logarithms of $\lambda$ and the scaling factor $\alpha$ defined in (3-1).

For functions of this form, the logarithm of the size of the projections on a space $V_{j}$ should decay linearly with $j$ irrespective of which wavelet is used. In particular, one does not need to use wavelets which are smoother than the regularity observed to obtain the scaling exponents, which also give the regularity.

In our numerical studies, we have used Daubechies wavelets of order 4,10 , and 20 , which we will denote as $\mathrm{D} 4, \mathrm{D} 10$, and $\mathrm{D} 20$, respectively. It is known that $\mathrm{D} 4 \in$ $\Lambda_{0.38 \ldots}$. For large $N, \mathrm{D} 2 N \in \Lambda_{l_{N}}$ where $l_{N} \approx 0.20775 N$. (See, e.g., [Härdle et al. 98, Sec. 7.1].)

We note that even if Theorem 5.10 does not apply to the measurements of regularity with D4 in some of the cases we consider, we obtain decays which are extremely similar to those obtained using D10 or D20, for which Theorem 5.10 does apply and also extremely similar to the regularities obtained by other methods. Moreover, we also note that the upper bounds given by Theorem 5.10 are identities.

We interpret the coincidence of the rates of decays obtained by any wavelets and the saturation of the bounds as (at least circumstantial) evidence that the the asymptotic scalings in (3-1) indeed hold. As we will discuss later, similar coincidences are observed for other methods.

In Figure 16, we show $\log _{2} \sup _{k}\left|\left\langle\theta, \psi_{j k}\right\rangle\right|$ vs. $j$ for several $\theta^{\mathrm{QN}}$ and $\theta^{\mathrm{NQ}}$ maps. The slope of the straight lines on this graph is $-\left(\alpha+\frac{1}{2}\right)$. There is one reason why this method works much better with wavelet instead of 


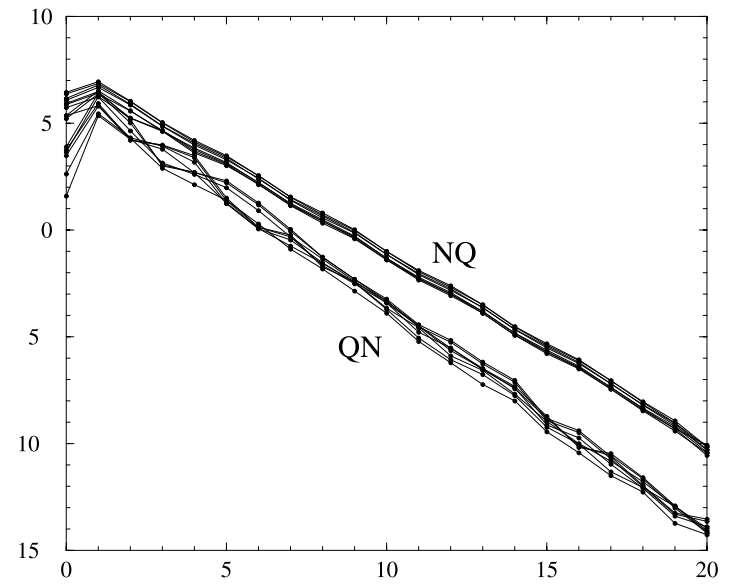

FIGURE 16. Plot of $\log _{2} \sup _{k}\left|\left\langle\theta, \psi_{j k}\right\rangle\right|$ vs. $j$ for 12 conjugacies of type NQ (for $2^{22}$ interpolated values based on $10^{7}$ iterates) and 12 of type QN (for $2^{21}$ interpolated values based on $10^{6}$ iterates).

Fourier coefficients (see Figure 10): The cubic interpolation in the large gaps distorts all Fourier coefficients. At the same time, in the case of wavelets, it only affects the ones whose support intersects the gap; moreover, the "artificial local smoothing" due to the interpolation decreases $\left|\left\langle\theta, \psi_{j k}\right\rangle\right|$ for the wavelets $\psi_{j k}$ supported at the gap, which does not change $\sup _{k}\left|\left\langle\theta, \psi_{j k}\right\rangle\right|$ for fixed $j$.

\subsection{Wavelet Approximation}

The method based on Theorem 5.11 yields very good results. Figure 17 shows plots of $\log _{2}\left\|\theta-\Pi_{j} \theta\right\|_{L^{\infty}(\mathbb{T})}$ vs. $j$ for several $\theta^{\mathrm{NC}}$ and $\theta^{\mathrm{CN}}$. The slope of the straight lines in this graph is $-\alpha$.

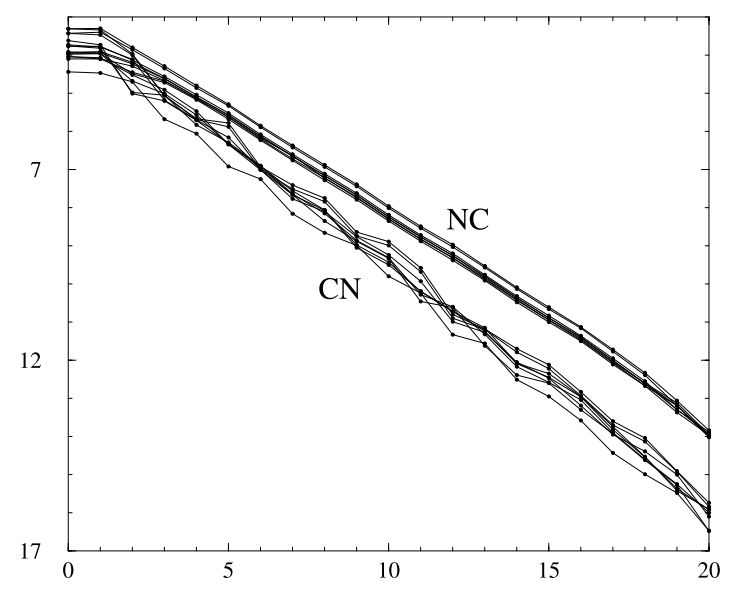

FIGURE 17. Plot of $\log _{2}\left\|\theta-\Pi_{j} \theta\right\|_{L^{\infty}(\mathbb{T})}$ vs. $j$ for 12 conjugacies of type $\mathrm{NC}$ (for $2^{22}$ interpolated values based on $10^{7}$ iterates) and 12 of type $\mathrm{CN}$ (for $2^{21}$ interpolated values based on $2 \times 10^{6}$ iterates).
As in the previous case, we note that we have used D4, D10 and D20. Theorem 5.11 does not apply to D4 in some cases. Nevertheless, we find the same linear decay as with the other methods and we interpret it as a confirmation of the asymptotic scaling of the function.

\section{RESULTS}

In this section, we give the numerical values of the Hölder exponents of the conjugacies. To determine these values, we used the methods based on Theorems 5.3, 5.8, 5.9, 5.10, and 5.11.

To find the smoothness of a particular type of conjugacy, we applied all these methods to study numerically the smoothness of the conjugacies between all possible combinations of circle maps studied (four N, four C, and four Q maps).

As an example, Table 3 shows the results of our analysis of the regularity of the conjugacies between $\mathrm{N}$ and $\mathrm{Q}$ maps as well as the results of the same methods applied to the test functions $w_{0.66745,3}$, whose Hölder exponent, $0.36800 \ldots$, is close to the one of the conjugacies of type $\theta^{\mathrm{NQ}}$.

The "Function" column indicates the function analyzed: " $w$ on $f_{0.2}^{\mathrm{N}}$ " means the regularity of the function $w_{0.66745,3}$ calculated at the points $\left(f_{0.2}^{\mathrm{N}}\right)^{n}(0)$, and $\theta\left(f_{0.2}^{\mathrm{N}} / f_{4 / 3}^{\mathrm{Q}}\right)$ means the conjugacy between $f_{0.2}^{\mathrm{N}}$ and $f_{4 / 3}^{\mathrm{Q}}$. The "Finite diffs" column shows the results of the smoothness found by using the finite difference method. The "CLP, $\eta=1,2,3$ " columns display the results of the CLP analysis for different numbers of derivatives. "Decay D4, D10, D20" contain the results of analysis of the decay rate of the coefficients of Daubechies 4 (resp. 10, 20) wavelets, while "Approx D4, D10, D20" shows the results of the study of the speed of the approximation using these wavelets. The meaning of the notation is the following: $0.3661(13)$ means $0.3661 \pm 0.0013$. The error is the standard error of the linear regression.

As seen in Table 3, the results obtained by using different methods are consistent, the most precise being the ones based on CLP. In Table 4, we give the Hölder exponent of the conjugacy between the maps $f$ and $g$. The margins of error are determined empirically, and only in very few cases are outliers ignored.

In the case of conjugacies of types $\mathrm{CC}$ and $\mathrm{QQ}$, for reasons explained in the text, we were not able to determine the smoothness of the conjugacies, but only to give rough estimates. 


\begin{tabular}{|c|c|c|c|c|c|c|c|c|c|c|}
\hline Function & Finite diffs & CLP, $\eta=1$ & CLP, $\eta=2$ & CLP, $\eta=3$ & Decay D4 & Decay D10 & Decay D20 & Approx D4 & Approx D10 & Approx D20 \\
\hline$w$ on $f_{0.2}^{\mathrm{N}}$ & $0.3508(162)$ & $0.3556(4)$ & $0.3661(13)$ & $0.3683(94)$ & $0.3548(126)$ & $0.3455(340)$ & $0.3558(461)$ & $0.3672(36)$ & $0.3658(102)$ & $0.3636(136)$ \\
\hline$w$ on $f_{0.3}^{\mathrm{N}}$ & $3511(156)$ & \begin{tabular}{|l|}
$0.3625(2)$ \\
\end{tabular} & $.3659(13)$ & $0.3680(93)$ & $0.3563(126)$ & $0.3460(350)$ & $0.3554(469)$ & $0.3685(38)$ & $0.3657(101)$ & $0.3650(141)$ \\
\hline$w$ on $f_{0.5}^{\mathrm{N}}$ & $0.3483(155)$ & $0.3632(2)$ & $0.3661(13)$ & $0.3681(94)$ & $0.3569(123)$ & $0.3478(344)$ & $0.3550(461)$ & $0.3659(32)$ & $0.3642(100)$ & $0.3645(140)$ \\
\hline$w$ on $f_{0.8}^{\mathrm{N}}$ & $0.3486(155)$ & \begin{tabular}{|l|}
$0.3634(4)$ \\
\end{tabular} & $0.3660(13)$ & $0.3682(94)$ & $0.3559(126)$ & $0.3482(345)$ & $0.3514(455)$ & $0.3678(33)$ & $0.3641(101)$ & $0.3620(138)$ \\
\hline$\theta\left(f_{0.2}^{\mathrm{N}} / f_{4 / 3}^{\mathrm{Q}}\right)$ & $0.3652(33)$ & $0.3611(10)$ & $0.3682(8)$ & $0.3676(2)$ & $0.3686(104)$ & $0.3667(247)$ & $0.3643(161)$ & $0.3713(12)$ & $0.3706(75)$ & $0.3664(69)$ \\
\hline$\theta\left(f_{0.2}^{\mathrm{N}} / f_{0.6}^{\mathrm{Q}}\right)$ & $0.3642(34)$ & $0.3622(12)$ & $0.3675(9)$ & $0.3710(6)$ & $0.3674(106)$ & $0.3556(160)$ & $0.3670(161)$ & $0.3717(11)$ & $0.3710(76)$ & $0.3660(72)$ \\
\hline$\theta\left(f_{0.2}^{\mathrm{N}} / f_{1.2}^{\mathrm{Q}}\right)$ & $0.3649(33)$ & $0.3613(10)$ & $0.3681(8)$ & $0.3670(3)$ & $0.3684(105)$ & $0.3670(249)$ & $0.3647(162)$ & $0.3711(12)$ & $0.3702(75)$ & $0.3661(70)$ \\
\hline$\theta\left(f_{0.2}^{\mathrm{N}} / f_{0.9}^{\mathrm{Q}}\right)$ & $0.3684(29)$ & $0.3623(11)$ & $0.3680(8)$ & $0.3667(3)$ & $0.3677(105)$ & $0.3744(217)$ & $0.3629(155)$ & $0.3710(13)$ & $0.3672(49)$ & $0.3658(70)$ \\
\hline$\theta\left(f_{0.3}^{\mathrm{N}} / f_{4 / 3}^{\mathrm{Q}}\right)$ & $0.3635(27)$ & $0.3616(10)$ & $0.3685(8)$ & $0.3677(3)$ & $0.3692(108)$ & $0.3658(246)$ & $0.3638(155)$ & \begin{tabular}{|l|}
$0.3701(8)$ \\
\end{tabular} & $0.3691(71)$ & $0.3669(67)$ \\
\hline$\theta\left(f_{0.3}^{\mathrm{N}} / f_{0.6}^{\mathrm{Q}}\right)$ & $0.3626(28)$ & $0.3625(12)$ & $0.3679(10)$ & $0.3703(5)$ & $0.3686(109)$ & $0.3632(270)$ & $0.3661(155)$ & \begin{tabular}{|l|}
$0.3703(7)$ \\
\end{tabular} & $0.3698(73)$ & $0.3665(68)$ \\
\hline$\theta\left(f_{0.3}^{\mathrm{N}} / f_{1.2}^{\mathrm{Q}}\right)$ & $0.3633(28)$ & $0.3618(10)$ & $0.3684(8)$ & $0.3671(3)$ & $0.3691(108)$ & $0.3695(234)$ & $0.3644(155)$ & $0.3698(8)$ & $0.3688(72)$ & $0.3666(67)$ \\
\hline$\theta\left(f_{0.3}^{\mathrm{N}} / f_{0.9}^{\mathrm{Q}}\right)$ & $0.3615(29)$ & $0.3628(11)$ & $0.3685(8)$ & $0.3668(3)$ & $0.3684(108)$ & $0.3546(345)$ & $0.3630(169)$ & $0.3700(8)$ & $0.3725(61)$ & $0.3665(67)$ \\
\hline$\theta\left(f_{0.5}^{\mathrm{N}} / f_{4 / 3}^{\mathrm{Q}}\right)$ & $0.3646(25)$ & $0.3610(10)$ & $0.3677(9)$ & $0.3671(2)$ & $0.3694(106)$ & $0.3631(249)$ & $0.3735(164)$ & $0.3712(9)$ & $0.3772(77)$ & $0.3728(70)$ \\
\hline$\theta\left(f_{0.5}^{\mathrm{N}} / f_{0.6}^{\mathrm{Q}}\right)$ & $0.3641(27)$ & $0.3616(11)$ & $0.3672(9)$ & $0.3684(3)$ & $0.3696(107)$ & $0.3641(195)$ & $0.3812(140)$ & $0.3712(9)$ & $0.3780(79)$ & $0.3724(72)$ \\
\hline$\theta\left(f_{0.5}^{\mathrm{N}} / f_{1.2}^{\mathrm{Q}}\right)$ & $0.3647(27)$ & $0.3612(10)$ & $0.3676(9)$ & $0.3666(3)$ & $0.3694(106)$ & $0.3663(216)$ & $0.3742(165)$ & \begin{tabular}{|l|}
$0.3710(9)$ \\
\end{tabular} & $0.3766(77)$ & $0.3725(70)$ \\
\hline$\theta\left(f_{0.5}^{\mathrm{N}} / f_{0.9}^{\mathrm{Q}}\right)$ & $0.3757(36)$ & $0.3620(10)$ & $0.3681(9)$ & $0.3664(3)$ & $0.3689(106)$ & $0.3642(308)$ & $0.3847(166)$ & $0.3709(10)$ & $0.3594(58)$ & $0.3723(70)$ \\
\hline$\theta\left(f_{0.8}^{\mathrm{N}} / f_{4 / 3}^{\mathrm{Q}}\right)$ & $0.3674(28)$ & $0.3607(10)$ & $0.3680(9)$ & $0.3654(4)$ & $0.3676(105)$ & $0.3682(249)$ & $0.3629(181)$ & $0.3684(7)$ & $0.3687(73)$ & $0.3692(66)$ \\
\hline$\theta\left(f_{0.8}^{\mathrm{N}} / f_{0.6}^{\mathrm{Q}}\right)$ & $0.3640(26)$ & $0.3615(10)$ & $0.3681(9)$ & $0.3645(3)$ & $0.3673(107)$ & $0.3701(239)$ & $0.3560(186)$ & $0.3685(7)$ & $0.3699(66)$ & $0.3695(68)$ \\
\hline$\theta\left(f_{0.8}^{\mathrm{N}} / f_{1.2}^{\mathrm{Q}}\right)$ & $0.3666(28)$ & $0.3612(10)$ & $0.3679(9)$ & $0.3649(5)$ & $0.3675(105)$ & $0.3681(250)$ & $0.3634(182)$ & $0.3683(7)$ & $0.3686(73)$ & $0.3691(66)$ \\
\hline$\theta\left(f_{0.8}^{\mathrm{N}} / f_{0.9}^{\mathrm{Q}}\right)$ & $0.3592(37)$ & $0.3624(10)$ & $0.3683(7)$ & $0.3649(5)$ & $0.3619(101)$ & $0.3355(325)$ & $0.3440(218)$ & $0.3687(7)$ & $0.3550(86)$ & $0.3694(66)$ \\
\hline
\end{tabular}

TABLE 3. Numerically-found regularity of $w_{0.66745,3}$ and all NQ conjugacies studied.

\begin{tabular}{|l|c|c|c|}
\hline$\downarrow f \quad g \rightarrow$ & $\mathrm{N}$ & $\mathrm{C}$ & $\mathrm{Q}$ \\
\hline $\mathrm{N}$ & Analytic & $0.527 \pm 0.003$ & $0.368 \pm 0.003$ \\
\hline $\mathrm{C}$ & $0.63 \pm 0.02$ & $1.4_{-0.2}^{+0.4}$ & $0.71 \pm 0.03$ \\
\hline $\mathrm{Q}$ & $0.54 \pm 0.05$ & $0.86 \pm 0.02$ & $1.7 \pm 0.5$ \\
\hline
\end{tabular}

TABLE 4. Regularity of the conjugacies.

\section{SOME BOUNDS ON THE REGULARITY OF CONJUGACIES}

\subsection{Some Simple Bounds}

It follows directly from the definition of $\Lambda_{\alpha}, 0<\alpha<1$, that if $h_{1} \in \Lambda_{\alpha_{1}}, h_{2} \in \Lambda_{\alpha_{2}}$, then $h_{1} \circ h_{2} \in \Lambda_{\alpha_{1} \alpha_{2}}$. It is not difficult to produce functions that satisfy the above bounds (just take $h_{i}(x)=|x|^{\alpha_{i}}$ ) as well as functions for which this bound is not optimal (take $h_{1}(x)=|x|^{\alpha_{1}}$, $\left.h_{2}=|x-0.1|^{\alpha_{2}}\right)$.

We also note that if $h^{1,2} \circ f_{1}=f_{2} \circ h^{1,2}, h^{2,3} \circ f_{2}=$ $f_{3} \circ h^{2,3}$, and we define $h^{1,3}$ by $h^{1,3}=h^{1,2} \circ h^{2,3}$, we have $h^{1,3} \circ f_{1}=f_{3} \circ h^{1,3}$.

Let $\rho^{a, b}$ (where $a, b$ are among N, C, Q) be the regularities of the conjugacy between golden mean circle maps of class $a$ to circle maps of class $b$, i.e., the entries in Table 4. It follows from the regularity of the composition that when $a, b, c$ are such that $a \neq b, b \neq c$, we should have

$$
\rho^{a, c} \geq \rho^{a, b} \rho^{b, c} .
$$

Inequality (8-1) can be verified in two cases in Table 4. Namely, we can take $a=\mathrm{N}, b=\mathrm{C}, c=\mathrm{Q}$, or $a=\mathrm{Q}$, $b=\mathrm{C}, c=\mathrm{N}$. When we carry out this verification, up to the error of the calculation, we find that (8-1) becomes an identity.

This is presumably not a coincidence. We believe that it is again a manifestation of the self-similarity of the function at small scales. If we compose two functions that, in each small scale, have oscillations comparable to those allowed by the Hölder exponent, the resulting function will also have oscillations that are comparable to the product of the Hölder exponents. Note, however, that this argument does not suggest that there is a simple relation between the regularity of a function and its inverse.

Equation (8-1) can be described by saying that the regularities of the conjugacies as indexed by the classes form a multiplicative supercocycle. We find empirically it is a cocycle.

\subsection{Scalings of the Recurrence and Upper Bounds on Hölder Exponents of Conjugacies}

Scalings have been studied numerically from the beginning of renormalization theory: some of them have been probed to hold.

In this section, we will report some rigorous results showing that if certain scalings hold, then there are bounds for the regularity of the conjugacy. Since these scaling relations - hypotheses of our lemma - are numerically accessible, we can use the rigorous results to obtain numerical upper bounds. 
One of the first numerical observations made in the study of the golden mean rotation number critical circle maps was that

$$
\left(f^{\bullet}\right)^{Q_{n}}(0) \approx \zeta_{\bullet}^{-n}
$$

where $\bullet$ stands for $\mathrm{N}, \mathrm{C}, \mathrm{Q}$, and $\zeta_{\bullet}$ are universal constants. The numbers $\zeta$ • play a fundamental role in the fixed point equations.

For noncritical maps, by Theorem $2.6, \zeta_{\mathrm{N}}$ is the same as for rotations by the golden mean, and, by using the well-known relation

$$
\frac{Q_{n}}{Q_{n+1}}=\gamma+C\left(-\gamma^{2}\right)^{n}+o\left(\gamma^{2 n}\right)
$$

we obtain $\zeta_{\mathrm{N}}=\gamma^{-1}$.

We note that for the cubic critical case, there are unpublished computer-assisted proofs ([Mestel 84], [Lanford and de la Llave 84]) that establish the existence of the $\zeta_{\mathrm{C}}$, upper and lower bounds for it, and the fact that (8-2) holds for maps in open sets.

Some relation between the scaling properties of the returns and the regularity of the conjugacy is given by the following lemma:

Lemma 8.1. Let

$$
\begin{aligned}
& f_{1}^{Q_{n}}(0)=C_{1} \zeta_{1}^{-n}+o\left(\zeta_{1}^{-n}\right) \\
& f_{2}^{Q_{n}}(0)=C_{2} \zeta_{2}^{-n}+o\left(\zeta_{2}^{-n}\right)
\end{aligned}
$$

and

$$
\alpha:=\log \left|\zeta_{2}\right| / \log \left|\zeta_{1}\right| \notin \mathbb{N} .
$$

If $h$ satisfies $h \circ f_{1}=f_{2} \circ h, h(0)=0$, then, for every $\delta>0, h \notin \Lambda_{\alpha+\delta}$.

Proof: For any $\chi>0$ we have

$$
\frac{h \circ f_{1}^{Q_{n}}(0)-h(0)}{\left[f_{1}^{Q_{n}}(0)-0\right]^{\chi}}=\frac{f_{2}^{Q_{n}}(0)}{\left[f_{1}^{Q_{n}}(0)\right]^{\chi}}=\frac{C_{2} \zeta_{2}^{-n}+o\left(\zeta_{2}^{-n}\right)}{C_{1}^{\chi} \zeta_{1}^{-\chi n}+o\left(\zeta_{1}^{-\chi n}\right)} .
$$

We argue by contradiction: if $h \in \Lambda_{\chi}$ for some $\chi>\alpha$, we use (8-6) to prove by induction that $h^{(n)}(0)=0$ for all $n \leq \alpha, n \in \mathbb{N}$. Then we note that $h \in \Lambda_{\alpha+\delta}$ (for any $\delta>0$ ) would imply that if we substitute $\chi=\alpha+\delta$ in (8-6), the lefthand side is bounded uniformly in $n$. At the same time, the righthand side of $(8-6)$ is unbounded in $n$.

We emphasize that Lemma 8.1 does not conclude anything when $\alpha \in \mathbb{N}$; in particular, it does not conclude anything in the cases when $f_{1}$ and $f_{2}$ have the same scaling factor $\zeta$, which happens when $f_{1}$ and $f_{2}$ are in the same universality class.

We have verified relations (8-2) for the maps we considered and obtained values of $\zeta$ as follows,

$$
\zeta_{\mathrm{C}}=-1.2886, \quad \zeta_{\mathrm{Q}}=-1.194,
$$

which agree with the values reported in [Shenker 82] (for the $\mathrm{C}$ case), and those in the papers [Hu et al. 90], [Delbourgo and Kenny 91], even though they consider functions that are periodized versions of polynomials which are not $C^{1}$.

Taking the values in (8-7) and the exact value for $\zeta_{N}$, we obtain the following upper bounds for the regularity of the conjugacies between the maps $f$ and $g$.

\begin{tabular}{|ll|c|c|c|}
\hline$\downarrow f$ & $g \rightarrow$ & $\mathrm{N}$ & $\mathrm{C}$ & $\mathrm{Q}$ \\
\hline $\mathrm{N}$ & & $?$ & 0.5269 & 0.368 \\
\hline $\mathrm{C}$ & & 1.898 & $?$ & 0.70 \\
\hline $\mathrm{Q}$ & & 2.72 & 1.43 & $?$ \\
\hline
\end{tabular}

TABLE 5. Upper bounds for the regularities of the conjugacies obtained from scaling of recurrences.

Note that in the cases NC, NQ, CQ, the upper bounds obtained by applying Lemma 8.1 agree, within the margin of error, with the values of the regularity reported. We conjecture that indeed the upper bounds produced by applying Lemma 8.1 for the other cases can not be interpreted as scalings at the moment.

The fact that the upper bounds that work in one case are far off in another seems to imply that there are different mechanisms limiting the regularity.

Since the $\alpha$ defined in (8-5) is the ratio of a quantity depending on the domain and a quantity depending on the range, the upper bounds that we have derived form a multiplicative cocycle.

For the sake of completeness, we also point out that there is a very similar argument that gives upper bounds for the regularity of the conjugacy for the maps in the same class. The following set of ideas was found useful in [de la Llave and Schafer, 96]. There it is shown that this argument produced upper bounds that were sharp in the case of conjugacies of limiting sets of unimodal maps.

Lemma 8.2. Assume that

$$
\begin{aligned}
& f_{1}^{Q_{n}}(0)=C_{1} \zeta^{-n}+D_{1} \tilde{\zeta}^{-n}+o\left(\tilde{\zeta}^{-n}\right) \\
& f_{2}^{Q_{n}}(0)=C_{2} \zeta^{-n}+D_{2} \tilde{\zeta}^{-n}+o\left(\tilde{\zeta}^{-n}\right)
\end{aligned}
$$

with $1<|\zeta|<|\tilde{\zeta}|, C_{1} C_{2} \neq 0$,

$$
D_{2} \neq\left(C_{2} / C_{1}\right) D_{1}
$$


and $\alpha:=\log |\tilde{\zeta}| / \log |\zeta| \notin \mathbb{N}$. Then, if $h$ satisfies $h \circ f_{1}=$ $f_{2} \circ h, h(0)=0$, then $h \notin \Lambda_{\alpha+\delta}$ for any $\delta>0$.

Proof: The argument is very similar to the argument that proved Lemma 8.1. Note that the assumptions imply that $\alpha>1$. Hence we are only excluding regularities higher than $C^{1}$.

We argue by contradiction, assuming that $h \in \Lambda_{\alpha+\delta}$. We note that

$$
h \circ f_{1}^{Q_{n}}(0)-h(0)=f_{2}^{Q_{n}}(0) .
$$

Since $h \in \Lambda_{\alpha+\delta}$, and $\alpha>1$, we conclude that $h^{\prime}(0)=$ $C_{2} / C_{1} \neq 0$. Hence $h$ is invertible in a neighborhood of zero and $h^{-1} \in \Lambda_{\alpha+\delta}$. We note that $h(t)=P(t)+R(t)$ where $P(t)$ is a polynomial of degree $[\alpha]$ and $|R(t)| \leq$ $|t|^{\alpha+\delta}$.

Given the assumptions on the degrees we have made, we have

$$
\begin{aligned}
& P\left(C_{1} \zeta^{-n}+D_{1} \tilde{\zeta}^{-n}+o\left(\tilde{\zeta}^{-n}\right)\right) \\
= & h^{\prime}(0) \tilde{\zeta}^{-n}+\sum_{j} C_{j}\left(\zeta^{j}\right)^{-n}+o\left(\zeta^{-n([\alpha]+1)}\right) .
\end{aligned}
$$

We also note that $R\left(C_{1} \zeta^{-n}+D_{1} \tilde{\zeta}^{-n}+o\left(\tilde{\zeta}^{-n}\right)\right)=o\left(\tilde{\zeta}^{-n}\right)$.

Equating the coefficients of the $\tilde{\zeta}^{-n}$ in (8-9), we obtain $D_{2}=\left(C_{2} / C_{1}\right) D_{1}$, which contradicts our assumption (8-8). This is the desired contradiction with the assumption that $h$ admitted a Taylor expansion near 0 with Hölder bounds.

Note that in contrast to Lemma 8.1, Lemma 8.2 contains a hypothesis (8-8) which could fail for a finite codimension set of maps. This is to be expected since the other hypothesis allows us to take $f_{1}=f_{2}, h=$ Id. In this situation, the conjugacy $h$ is very regular. The elementary theory of renormalization tells us that (8-8) occurs in a set which is of positive codimension.

Conjecture 3.1 amounts to the fact that the upper bounds produced by applying Lemma 8.2 are sharp.

At the moment, unfortunately, we do not have accurate enough values for the $\tilde{\zeta}$ for cubic critical or quintic critical maps and, hence, cannot give concrete values for the upper bounds of the regularity in the CC or QQ cases. It is interesting to remark that, in the NN case, these bounds are far from optimal.

\section{CONCLUSION}

We have studied the smoothness of the conjugacies between circle maps with critical points and with golden mean rotation number. The first step was to obtain an extremely precise calculation of the parameters of the function that put us in the correct universality class. The most important step was to develop a numerical toolkit based on a wide array of methods from harmonic analysis (Littlewood-Paley, wavelets) to study the regularity and the fine scale structure of these functions. We have used the combination of the methods applied to critical circle maps to asses their range of validity, and have found indication that the regularity of critical circle maps is possibly limited by considerations other than just scaling of recurrences.

\section{ACKNOWLEDGMENTS}

We are grateful to John Gilbert, Álex Haro, Ricardo PérezMarco, Marek Rychlik and John Vano for fruitful discussions. We would also like to thank some people we do not know in person, but who generously made their software products freely available. We thank Paul J. Turner for the plotting tool ACE/gr, Keith Briggs for the doubledouble routines, and the team developing the library for arbitrary precision arithmetic GNU MP. Of course, we have relied on the work of many other people included in the references. We have developed and run our programs on the computers of the Department of Mathematics of the University of Texas at Austin. Our research was partially supported by NSF and TARP grants.

\section{REFERENCES}

[Arneodo and Holschneider 88] A. Arneodo and M. Holschneider. "Fractal dimensions and homeomorphic conjugacies," J. Statist. Phys. 50:5-6 (1988), 995-1020.

[Arnold 61] V. I. Arnol'd. "Small denominators. I. mappings of the circumference onto itself," Izv. Akad. Nauk SSSR Ser. Mat. 25 (1961), 21-86, correction: 28 (1964), 479480. English translation: Amer. Math. Soc. Transl. (2) 46 (1965), 213-284.

[Berggren et al. 00] L. Berggren, J. Borwein, and P. Borwein. Pi: A Source Book, Second Ed., Springer, New York, 2000.

[Bohr et al. 84] T. Bohr, P. Bak, and M. H. Jensen. "Transition to chaos by interaction of resonances in dissipative systems. II. Josephson Junctions, Charge-Density Waves, and Standard Maps," Phys. Rev. A (3) 30:4 (1984), 1970-1981.

[Borwein and Borwein 84] J. M. Borwein and P. B. Borwein. "The arithmetic-geometric mean and fast computation of elementary functions," SIAM Rev. 26:3 (1984), 351366.

[Borwein and Borwein 87] J. M. Borwein and P. B. Borwein. $P i$ and the AGM: A study in analytic number theory and computational complexity, Wiley, New York, 1987. 
[Brent 76] R. P. Brent. "Fast multiple-precision evaluation of elementary functions," J. Assoc. Comput. Mach. 23:2 (1976), 242-251.

[Briggs 00] K. M. Briggs. The doubledouble homepage. www.labs.bt.com/people/briggsk2/doubledouble.html.

[Briggs at al. 98] K. M. Briggs, T. M. Dixon, and G. Szekeres. "Analytic solutions of the Cvitanović-Feigenbaum and Feigenbaum-Kadanoff-Shenker equations," Internat. J. Bifur. Chaos Appl. Sci. Engrg. 8:2 (1998), 347-357.

[Cvitanović 89] P. Cvitanović (Ed.) Universality in Chaos, Second ed., Adam Hilger, Bristol, 1989.

[Daubechies 92] I. Daubechies. Ten Lectures on Wavelets, SIAM, Philadelphia, PA, 1992.

[Denjoy 32] A. Denjoy. "Sur les courbes définies par les équations différentielles à la surface du tore," J. Math. Pures et Appl. (9. série) 11 (1932), 333-375.

[Dixon et al. 97] T. M. Dixon, B. G. Kenny, and K. M. Briggs. "On the universality of singular circle maps," Phys. Lett. A 231:5-6 (1997), 359-366.

[Delbourgo and Kenny 91] R. Delbourgo and B. G. Kenny. "Relations between universal scaling constants for the circle map near the golden mean," J. Math. Phys. 32:4 (1991), 1045-1051.

[Eckmann and Epstein 86] J. P. Eckmann and H. Epstein. "On the existence of fixed points of the composition operator for circle maps," Comm. Math. Phys. 107:2 (1986), 213-231.

[Eckmann and Epstein 87] J.-P. Eckmann and H. Epstein "Fixed points of composition operators." In VIIIth International Congress on Mathematical Physics (Marseille, 1986), pp. 517-530, World Scientific, Singapore, 1987.

[Epstein 86] H. Epstein. "New proofs of the existence of the Feigenbaum functions," Comm. Math. Phys. 106:3 (1986), 395-426.

[Epstein 89] H. Epstein. "Fixed points of composition operators. II," Nonlinearity 2:2 (1989), 305-310.

[Feigenbaum et al. 82] M. J. Feigenbaum, L. P. Kadanoff, and S. J. Shenker. "Quasiperiodicity in dissipative systems: a renormalization group analysis," Physica 5D:2-3 (1982), 370-386.

[de Faria 99] E. de Faria, "Asymptotic rigidity of scaling ratios for critical circle mappings," Ergodic Theory Dynam. Systems 19:4 (1999), 995-1035.

[de Faria and de Melo 99] E. de Faria and W. de Melo. "Rigidity of critical circle mappings. I," J. Eur. Math. Soc. (JEMS) 1:4 (1999), 339-392.

[de Faria and de Melo 00] E. de Faria and W. de Melo. "Rigidity of critical circle mappings. II," J. Amer. Math. Soc. 13:2 (2000), 343-370.

[Forsythe et al. 77] G. E. Forsythe, M. A. Malcolm, and C. B. Moller. Computer Methods for Mathematical Computations, Prentice-Hall, Englewood Cliffs, 1977.
[Frazier et al. 91] M. Frazier, B. Jawerth, and G. Weiss. Littlewood-Paley Theory and the Study of Function Spaces, AMS, Providence, RI, 1991.

[Ghering and Palka, xx] F. W. Ghering and B. Palka. QuasiConformal Maps Manuscript.

[Glass 95] L.Glass. "Cardiac arrhythmias and circle maps - a classical problem," Internat. J. Bifur. Chaos Appl. Sci. Engrg. 5 (1995), 359-371.

[GMP 00] The GMP home page. www.swox.com/gmp/.

[Greene 79] J. M. Greene. "A method for determining a stochastic transition," J. Math. Phys. 20:6 (1979), 11831201.

[Hall 81] G. R Hall. "A $C^{\infty}$ Denjoy counterexample," Ergodic Theory Dynamical Systems 1:3 (1981), 261-272.

[Härdle et al. 98] W. Härdle, G. Kerkyacharian, D. Picard, and A. Tsybakov. Wavelets, Approximation, and Statistical Applications, Springer-Verlag, New York, 1998.

[Bai-Lin Hao 90] Bai-Lin Hao (Ed.) Chaos II, World Scientific, Singapore, 1990.

[Herman 79] M. R. Herman. "Sur la conjugaison différentiable des difféomorphismes du cercle à des rotations," Inst. Hautes Études Sci. Publ. Math. No. 49 (1979), 5-233.

[Herman 83] M. R. Herman. "Sur les difféomorphismes du cercle de nombre de rotation de type constant." In Conference on Harmonic Analysis in Honor of Antoni Zygmund, (Chicago, Ill., 1981), pp. 708-725, Wadsworth, Belmont, CA, 1983.

[Hernández and Weiss 96] E. Hernández and G. Weiss. $A$ First Course on Wavelets, CRC Press, Boca Raton, FL, 1996.

[Holschneider and Tchamitchian 91] M. Holschneider and $\mathrm{Ph}$. Tchamitchian. "Pointwise analysis of Riemann's "nondifferentiable" function," Invent. Math. 105 (1991), 157-175.

[Hu et al. 90] B. Hu, A. Valinia, and O. Piro. "Universality and asymptotic limits of the scaling exponents in circle maps," Phys. Lett. A 144:1 (1990), 7-10.

[Hu and Sullivan 97] J. Hu and D. P. Sullivan. "Topological conjugacy of circle diffeomorphisms," Ergodic Theory Dynam. Systems 17:1 (1997), 173-186.

[Ignatov 95] A. M. Ignatov. "Trivelpiece-Gould modes in a corrugated plasma slab," Phys. Rev. E 51:2 (1995), 1391-1399.

[Jaffard 97] S. Jaffard. "Multifractal formalism for functions. I. Results valid for all functions. II. Self-similar functions," SIAM J. Math. Anal. 28:4 (1997), 944-970, 971998. 
[Jaffard and Meyer 96] S. Jaffard and Y. Meyer. "Wavelet methods for pointwise regularity and local oscillations of functions," Mem. Amer. Math. Soc. 123 (1996), no. 587.

[Katok and Hasselblatt 95] A. Katok and B. Hasselblatt. Introduction to the Modern Theory of Dynamical Systems, Cambridge University Press, Cambridge, 1995.

[Katznelson and Ornstein 89] Y. Katznelson and D. Ornstein. "The differentiability of the conjugation of certain diffeomorphisms of the circle," Ergodic Theory Dynamical Systems 9:4 (1989), 643-680.

[Kim and Ostlund 89] S. Kim and S. Ostlund. "Universal scaling in circle maps," Physica 39D:2-3 (1989), 365392.

[Krantz 83] S. G. Krantz. "Lipschitz spaces, smoothness of functions, and approximation theory," Exposition. Math. 1:3 (1983), 193-260.

[Lanford 86] O. E. Lanford III. "Renormalization group methods for circle mappings." In Statistical Mechanics and Field Theory: Mathematical Aspects (Groningen, 1985), pp. 176-189, Lecture Notes in Phys., 257, Springer, Berlin, 1986.

[Lanford 87] O. E. Lanford III. "Renormalization group methods for critical circle mappings with general rotation number." In VIIIth International Congress on Mathematical Physics (Marseille, 1986), pp. 532-536, World Scientific, Singapore, 1987.

[Lanford 88] O. E. Lanford III. "Renormalization group methods for circle mappings." In Nonlinear Evolution and Chaotic Phenomena (Noto, 1987), pp. 25-36 NATO Adv. Sci. Inst. Ser. B Phys., 176, Plenum, New York, 1988.

[Lanford and de la Llave 84] O. E. Lanford III and R. de la Llave. "Solution of the functional equation for critical circle mappings with golden rotation number." Manuscript (1984).

[de la Llave and Petrov 99] R. de la Llave and N. Petrov. "Theory of circle maps and the problem of onedimensional optical resonator with a periodically moving wall," Phys. Rev. E (3) 59:6 (1999), 6637-6651.

[de la Llave and Schafer, 96] R. de la Llave and R. P. Schafer. "Rigidity properties of one dimensional expanding maps and applications to renormalization." Manuscript, 1996.

[Louis et al. 97] A. K. Louis, P. Maaß, and A. Rieder. Wavelets: Theory and Applications, Wiley, Chichester, 1997.

[Mallat 98] S. Mallat. A Wavelet Tour of Signal Processing, Academic Press, San Diego, CA, 1998.

[de Melo 98] W. de Melo. "Rigidity and renormalization in one-dimensional dynamical systems." In Proceedings of the International Congress of Mathematicians, Vol. II (Berlin, 1998), pp. 765-778 Doc. Math. 1998, Extra Vol. II (electronic).

[de Melo and Van Strien 98] W. de Melo and S. Van Strien. One-Dimensional Dynamics, Springer, Berlin, 1993.
[Mestel 84] B. Mestel. Univ. of Warwick thesis, (1984).

[Meyer 98] Y. Meyer. Wavelets, Vibrations and Scalings, AMS, Providence, RI, 1998.

[Meyer 90] Y. Meyer Ondelettes et Opérateurs. I, Hermann, Paris, 1990.

[Moser 66] J. Moser. "A rapid convergent iteration method and non-linear differential equations. II." Ann. Scuola Norm. Sup. Pisa (3) 20 (1966), 499-535.

[Moser and Pöschel 84] J. Moser and J. Pöschel. "An extension of a result by Dinaburg and Sinai on quasiperiodic potentials," Comment. Math. Helv. 59:1 (1984), 39-85.

[Ostlund et al. 83] S. Ostlund, D. Rand, J. Sethna, and E. Siggia. "Universal properties of the transition from quasiperiodicity to chaos in dissipative systems," Physica 8D:3 (1983), 303-342.

[Pinto and Rand 92] A. A. Pinto and D. A. Rand. "Global phase space universality, smooth conjugacies and renormalization. II. The $C^{k+\alpha}$ case using rapid convergence of Markov families," Nonlinearity 5:1 (1992), 49-79.

[Poincaré 1885] H. Poincaré. "Sur le courbes définies par les équations différentielles," J. Math Pures et Appl. (4. série)," 1 (1885), 167-244. Reprinted in Euvres de Henri Poincaré, Tome I, Gauthier-Villars, Paris, 1928.

[Pommeau and Manneville 80] Y. Pommeau and P. Manneville. "Intermittent transition to turbulence in dissipative dynamical systems," Comm. Math. Phys. 74:2 (1980), 189-197.

[Press et al. 92] W. H. Press, S. A. Teukolsky, W. T. Vetterling, and B. P. Flannery. Numerical Recipes in $C$, Second ed., Cambridge University Press, Cambridge, 1992. Also available at http://www.nr.com/.

[Rand 87] D. A. Rand. "Fractal bifurcation sets, renormalization strange sets and their universal invariants," Proc. $R$. Soc. Lond. A 413 (1987), 45-61.

[Rand 88a] D. A. Rand. "Global phase space universality, smooth conjugacies and renormalisation. I. The $C^{1+\alpha}$ case," Nonlinearity 1:1 (1988), 181-202.

[Rand 88b] D. Rand. "Universality and renormalisation in dynamical systems." In New Directions in Dynamical Systems, pp. 1-56, Cambridge Univ. Press, Cambridge, 1988 .

[Rand 92] D. A. Rand. "Existence, nonexistence and universal breakdown of dissipative golden invariant tori. I. Golden critical circle maps," Nonlinearity 5:3 (1992), 639-662.

[Rand et al. 82] D. Rand, S. Ostlund, J. Sethna, and E. D. Siggia. "Universal transition from quasiperiodicity to chaos in dissipative systems," Phys. Rev. Lett. 49:2 (1982), 132-135.

[Shenker 82] S. Shenker. "Scaling behavior in a map of a circle onto itself: empirical results," Physica 5D:2-3 (1982), $405-411$. 
[Shraiman 84] B. I. Shraiman. "Transition from quasiperiodicity to chaos: a perturbative renormalization-group approach," Phys. Rev. A (3) 29:6 (1984), 3464-3466.

[Sinai and Khanin 89] Ya. G. Sinai and K. M. Khanin. "Smoothness of conjugacies of diffeomorphisms of the circle with rotations," Uspekhi Mat. Nauk 44:1(265) (1989), 57-82, 247. English translation: Russian Math. Surveys 44:1 (1989), 69-99.

[Stein 70] E. Stein. Singular Integrals and Differentiability Properties of Functions, Princeton University Press, Princeton, 1970.

[Świątek 91] G. Świątek. "Circle homeomorphisms with flat critical points," Fund. Math. 138:3 (1991), 205-217.

[Świątek 98] G. Świątek. "On critical circle homeomorphisms," Bol. Soc. Brasil. Mat. (N.S.) 29:2 (1998), 329351.

[Tangerman and Veerman 91] F. M. Tangerman and J. J. P. Veerman. "Scalings in circle maps. II," Comm. Math. Phys. 141:2 (1991) 279-291.
[Väisälä 71] J. Väisälä. Lectures on n-Dimensional Quasiconformal Mappings (Lecture Notes in Mathematics, Vol. 229), Springer, Berlin, 1971.

[Veerman and Tangerman 90] J. J. P. Veerman and F. M. Tangerman. "Scalings in circle maps. I," Comm. Math. Phys. 134:1 (1990), 89-107.

[Wickerhauser 94] M. V. Wickerhauser. Adapted Wavelet Analysis from Theory to Software, A K Peters, Wellesley, MA, 1994. Software available at http://www. math. wustl. edu/ victor/.

[Yampolsky 99] M. Yampolsky. "Complex bounds for renormalization of critical circle maps," Ergodic Theory Dynamical Systems 19:1 (1999), 227-257.

[Yoccoz 84a] J.-C. Yoccoz. "Il n'y a pas de contre-exemple de Denjoy analytique," C. R. Acad Sc. Paris Sér. I Math. 298:7 (1984), 141-144.

[Yoccoz 84b] J.-C. Yoccoz. "Conjugaison différentiable des difféomorphismes du cercle dont le nombre de rotation vérifie une condition diophantienne," Ann. Sci. École Norm. Sup. (4) 17:3 (1984), 333-359.

Rafael de la Llave, Department of Mathematics, University of Texas at Austin, Austin, TX 78712 (llave@math.utexas.edu)

Nikola P. Petrov, Department of Mathematics, University of Michigan, Ann Arbor, MI 48109 (npetrov@umich.edu)

Received May 10, 2001; accepted November 2, 2001. 